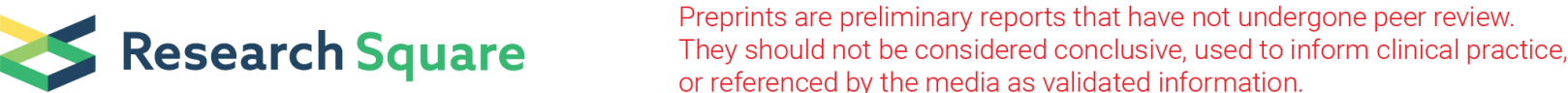

\section{A Single-Cell Immune Atlas of Triple Negative Breast Cancer Reveals Novel Immune Cell Subsets}

\section{Si Qiu}

Sun Yat-sen University Cancer Center, State Key Laboratory of Oncology in South China, Collaborative Innovation Center of Cancer Medicine

\section{Ruoxi Hong}

Sun Yat-sen University Cancer Center, State Key Laboratory of Oncology in South China, Collaborative Innovation Center of Cancer Medicine

\section{Zhenkun Zhuang}

BGI-Shenzhen

Linnan Zhu

BGI-Shenzhen

Yuan Li

Sun Yat-sen University Cancer Center

\section{Weimin Zhang}

Cancer Institute and Cancer Hospital, Chinese Academy of Medical Sciences and Peking Union Medical College

Xinxin Lin

BGI-Shenzhen

\section{Qiufan Zheng}

Sun Yat-sen University Cancer Center https://orcid.org/0000-0003-3752-8506

\section{Shang Liu}

BGI-Shenzhen

\section{Kai Zhang}

Sun Yat-sen University Cancer Center

\section{Mengxian Huang}

BGI-Shenzhen, Shenzhen 518103, China

\section{Kaping Lee}

Sun Yat-sen University Cancer Center, Guangzhou 510060, China

\section{Qianyi Lu}

Sun Yat-sen University Cancer Center, Guangzhou 510060, China

\section{Wen Xia}

Sun Yat-sen University Cancer Center, Guangzhou 510060, China

\section{Fei Xu}


Sun Yat-sen University Cancer Center, Guangzhou 510060, China https://orcid.org/0000-0001-85436636

\section{Yong Hou}

BGI-Shenzhen, Shenzhen 518103, China

\section{Xiuqing Zhang}

BGI-Shenzhen, Shenzhen 518103, China

\section{Jian Wang}

BGI-Shenzhen, Shenzhen 518103, China

\section{Huanming Yang}

BGI-Shenzhen, Shenzhen 518103, China

\section{Qimin Zhan}

Key laboratory of Carcinogenesis and Translational Research (Ministry of Education/Beijing), Laboratory of Molecular Oncology, Peking University Cancer Hospital \& Institute, Beijing 100142, China

\section{Bo Li}

BGI-Genolmmune, BGI-Shenzhen, Wuhan 4300794, China

\section{Shusen Wang ( $\square$ wangshs@sysucc.org.cn )}

Sun Yat-sen University Cancer Center

\section{Article}

Keywords: triple-negative breast cancer, immune landscape, single-cell RNA sequencing

Posted Date: September 2nd, 2020

DOI: https://doi.org/10.21203/rs.3.rs-64982/v1

License: (c) (1) This work is licensed under a Creative Commons Attribution 4.0 International License. Read Full License 


\section{Abstract}

Background: Triple-negative breast cancer (TNBC) represents the most aggressive breast cancer subtype, which recently attracts great interest for immune therapeutic development. In this context, in-depth understanding of TNBC immune landscape is highly demanded.

Results: Here we report full-length single-cell RNA sequencing results of 9683 tumor-infiltrated immune cells isolated from 14 treatment naïve TNBC tumors, where 22 immune cell subsets, including T cells, macrophages, $B$ cells, and DCs have been characterized. We identify a new $T$ cell subset, CD $8^{+} \mathrm{CXCL} 8^{+} \mathrm{T}$ cell, which associates with poor survival, and a subset of "pre-exhaustion" $T$ cell cluster, which is predictive of favorable prognosis. A novel immune cell subset comprised of $\mathrm{TCR}^{+}$macrophages, is found to be widely distributed in TNBC tumors. Further analyses reveal an up-regulation of molecules associated with TCR signaling and cytotoxicity in these immune cells.

Conclusions: Altogether, our study provides a valuable resource to understand the immune ecosystem of TNBC. The novel immune cell subsets reported herein might be functionally important in cancer immunity. These data will be helpful for the immunotherapeutic strategy design of this disease.

\section{Background}

Cancer immunotherapies are revolutionizing cancer treatment landscape. Current checkpoint blockade therapies mainly function to rescue $T$ cells from exhaustion or eliminate $T$ regulatory cells (Treg). Emerging evidence indicates that myeloid cells, such as macrophages, are highly influential on other cell populations in the tumor microenvironment (TME), including cancer cells as well as immune cells $1,2,3$. Whereas most myeloid cells promote cancer outgrowth, others display potent anti-tumour activity ${ }^{3}$. Targeting the myeloid cell population has attracted increasing interest in recent years ${ }^{4,5}$. While checkpoint blockade therapies have demonstrated remarkable clinical activity in many cancer types, the biological determinants of response to these agents remain incompletely understood. Several factors such as tumor neoantigens, mutation burden, tumor-infiltrating lymphocytes (TILs) levels, and PD-L1 expression have shown correlation with response, but other factors from the microenvironment also have profound influences ${ }^{6}$. The design of novel cancer immunotherapy strategies and the identification of effective clinical biomarkers require deep understanding of this ecosystem.

T cells are one of the key component and best-characterized population in the $\operatorname{TME}$ of solid tumors ${ }^{7,8}$. $\mathrm{CD} 4^{+}$helper $\mathrm{T}$ cells and $\mathrm{CD} 8^{+}$cytotoxic $\mathrm{T}$ cells can exert anti-tumor effect by targeting antigenic tumor cells, and levels of activated $C D 8^{+} T$ cells are predictive of good prognosis in several cancers ${ }^{9,10}$. However, the tumor microenvironment can develop various mechanisms to suppress $T$ cell responses and facilitate cancer cell survival. These mechanisms may involve Tregs, which secrete immunosuppressive cytokines, and myeloid and stromal cells, which modulate immune check points by activation of coinhibitory receptors (e.g., PD-1, Tim-3, and CTLA-4) on T cells, driving T cell dysfunction and exhaustion 11,12. Understanding the mechanisms of TME induced T-cell dysfunction should assist on the 
development of promising combinatorial immunotherapies to improve the clinical efficacy of current immune checkpoint blockades.

Tumor-associated macrophages (TAMs) are another key component of the TME with a dual supportive and inhibitory influence on cancer growth ${ }^{13}, 14$. Customized functional model divides macrophages into to two categories: classical M1 and alternative M2 macrophages. The M1 macrophage is involved in the inflammatory response, pathogen clearance, and antitumor immunity. In contrast, the M2 macrophage influences an anti-inflammatory response, wound healing, and pro-tumorigenic properties ${ }^{15}$. TAM phenotypes are highly plastic, and recent reports show that the model distinguishing between classically polarized anti-tumor M1 and alternatively polarized pro-tumor M2 subtypes incompletely accounts for the phenotypic diversity in vivo ${ }^{16}$. Azizi et al found that M1 and M2 gene signatures are positively correlated in the myeloid populations in breast cancer tumors ${ }^{17}$.

Triple-negative breast cancer (TNBC) represents up to $20 \%$ of all breast cancers. TNBC tumors are typically more aggressive and difficult to treat than hormone receptor-positive tumors, and are associated with a higher risk of early relapse. The lack of estrogen receptor, progesterone receptor, and HER2 expression precludes the use of targeted therapies, and the only approved systemic treatment option is chemotherapy. Responses to chemotherapy occur, but are often short lived and frequently accompanied by considerable toxicity. Gene profiling studies reveal that TNBCs are highly heterogeneous and a large proportion of them demonstrate DNA Repair Deficiency Signature $18,19,20$. Recent data showed impressive activity of PD-1/PD-L1 blockade therapy in metastatic TNBC patients who were chemotherapy naïve, suggesting early intervention of immunotherapy can bring more benefit ${ }^{21}$. Clinical trials applying checkpoint inhibitors in the neo-adjuvant setting of TNBC are ongoing. Although it needs to be confirmed in a larger cohort, these results are consistent with the notion that immunotherapy agents are most

efficient at low tumor burden and in patients naïve of immune-modulatory chemotherapy agents ${ }^{22,23}$. To better understand the immune ecosystem of TNBC, we analyzed the full-length single-cell RNA sequencing data of 9,683 tumor-infiltrated immune cells isolated from treatment naïve TNBC tumors. We identified 22 unique immune cell subsets, including T cells, macrophages, B cells, and DCs. Using combined expression and TCR-based analyses, we were able to indicate the function and developmental path of $\mathrm{T}$ cell subsets. We found a novel $\mathrm{T}$ cell subset, $\mathrm{CD} 8^{+} \mathrm{CXCL} 8^{+} \mathrm{T}$ cell, and revealed its prognostic value. We also demonstrated the co-expression pattern of M1/M2 signature on single-cell macrophages and revealed the wide existence of $\mathrm{TCR}^{+}$macrophages. This is the first time that $\mathrm{TCR}^{+}$macrophages were studied on single-cell transcriptome level.

\section{Results}

\section{Tumor Characteristics and Single-cell Transcriptome Profiling of Immune Cells}

To uncover the complexity of immune ecosystem in TNBC, we performed deep single-cell RNA sequencing on immune cells flow-sorted from the primary tumors of 14 treatment naïve TNBC patients 
based on the expression of CD45 (Fig. 1a and Supplementary Fig. 1a). After filtering out cells with low quality, we obtained $9,683 \mathrm{CD} 45^{+}$cells with an average of 14.80 million uniquely mapped reads per cell (Supplementary Fig. 1b and Supplementary Table 1). This sequencing depth assured the detection for genes with low expression level, allowing reliably profiling of cytokines and transcription factors in immune cells (Supplementary Fig. 1c).

\section{The Immune Landscape of TNBC}

To reveal the immune cell populations in TNBC, we performed unsupervised clustering using Seruat method on $\mathrm{CD}_{4} 5^{+}$cells and obtained 22 cell clusters, which were then visualized by t-SNE algorithm ${ }^{24}$ (Fig. 1b). We identified the immune cells as T cells, B cells, macrophages and DCs based on the expression of classic cell type markers as well as reference component analysis ${ }^{25}$ (Fig. $1 \mathrm{c}$ and Supplementary Fig. 1d). With this approach, nine clusters were annotated as $\mathrm{T}$ cell clusters, six as macrophage clusters, three as B cell clusters, two as DC clusters, and also there remained two clusters that could not be well determined (Fig. 1b and Supplementary Fig. 1e). The two DC clusters could be further categorized as one classical DC subset that expressed CD1C and Dectin 1 (CLEC7A), and one plasmacytoid DC subset specifically expressing IL3RA and CD303 $(C L E C 4 C)^{26}$. For the B cell clusters, all three clusters are $C D 10^{-}$mature $B$ cells ${ }^{27}$. Most of the clusters consisted of cells from multiple patients, indicative of common immune traits among patients (Fig. 1d; Supplementary Fig. 1f). Besides, the proportion of different immune cell types and clusters varied across patients, consistent with descriptions in previous researches (Fig. 1e,f; Supplementary Fig. 1g) ${ }^{17}$.

\section{Subtype Analysis of Tumor Infiltrated T cells}

Next, we sought to characterize infiltrated T cells in TNBC tumors. Unsupervised clustering of CD $45^{+}$cells identified nine $\mathrm{T}$ cell clusters, including three clusters of $\mathrm{CD} 4^{+} \mathrm{T}$ cells, and six clusters of $\mathrm{CD} 8^{+} \mathrm{T}$ cells (Fig. 2a). Distinct signatures of $T$ cell clusters were revealed by expression patterns of known $T$ cell functional markers (cytotoxic, naïve, regulatory or exhausted) and differentially expressed genes (DEGs) among clusters (Fig. 2b, c; and Supplementary Table 2). Among the CD8 ${ }^{+}$clusters, $\mathrm{T} 2$ and T4 were suggested as $\mathrm{CCR}^{+} \mathrm{T}$ cell clusters for their specific expression of $C C R 7, S E L L$, and $L E F 1$, indicative of naïve or memory $T$ cell ${ }^{28}$. To reveal the proportion of naïve $T$ cells in $T 2$ and $T 4$, we analyzed the expression of CD45RA/RO isoforms (method). We showed that the proportions of CD45RA, which indicates T cells' naïve status, were $14.2 \%$ and $8.0 \%$ for T2 and T4, respectively. This observation was further confirmed by flow cytometry analyses on cells isolated from three freshly collected TNBC samples, using antibodies of CD45RA, CD45RO and CD8. We found that CD45RA ${ }^{+} \mathrm{CD}^{+}$cells accounted for $6.33 \%, 8.27 \%$ and $17.46 \%$ of $\mathrm{CD}^{+}{ }^{+}$cells, respectively (Supplementary Fig. 2). These results indicated that naïve $\mathrm{T}$ cells accounted for a small portion of T2 and T4, others were considered as memory T cells. T1 and T6 showed high expression of exhaustion markers HAVCR2 (TIM-3), TIGIT, and $L A G 3^{29}$, suggestive of exhausted CD ${ }^{+} T$ cell clusters. T3 and T5 were characterized by high expression of genes associated with cytotoxicity, 
including IFNG, PRF1, GZMA and GZMB, and meanwhile expressed low levels of exhaustion markers, representing cytotoxic $T$ cell clusters (Fig. $2 b$ ).

For the three $\mathrm{CD}^{+}$clusters, $\mathrm{T} 7$ and $\mathrm{T} 9$ exhibited remarkable regulatory $\mathrm{T}$ cell (Treg) features for high expression of FOXP3 and IL2RA (CD25). The remaining $C D 4^{+}$cluster T8 was marked by high levels of CXCL13, CD200, BTLA, PDCD1 (PD-1), and CTLA4, consistent with previously reported dysfunctional T helper cell (Th) features ${ }^{30,31}$. Both $C D 8^{+}$and $C D 4^{+} T$ cell clusters exhibited distinct distributions among patients (Fig. 2d).

\section{Genes Uniquely Expressed by Exhausted CD8 ${ }^{+} \mathrm{T}$ Cells and Tumor-infiltrated Tregs in TNBC}

Current immune checkpoint blockade therapies mainly target exhausted CTL or Tregs. To uncover potential therapeutic targets for immunotherapy of TNBC, we sought to investigate genes uniquely expressed by these two immunosuppressive T cell subsets. By comparing the expression profiles of exhausted $C D 8^{+}$clusters (T1 and T6) with non-exhausted clusters (T2, T3, T4 and T5) using R package limma, we obtained a set of 114 exhaustion-specific genes (adjusted $P<0.01$ and $\log _{2} F C \geq 1$, Supplementary Table 3), including multiple known exhaustion markers, such as TIGIT, HAVCR2, LAG3, and $C T L A 4^{12}$ (Fig. 2e). Among these genes, 23 were also demonstrated in the exhausted $\mathrm{CD}^{+}{ }^{+}$cellspecific gene list by a previous study of liver cancer ${ }^{31}$ (Fig. 2e; Supplementary Table 4). Notably, tumorinfiltrating $\mathrm{CD}^{+}{ }^{+}$cells in our TNBC tumors expressed low level of PDCD1 (PD-1), while high levels of other exhaustion markers such as HAVCR2, TIGIT, and CTLA4 were detected (Fig. 2g). While both exhausted T cell clusters showed high levels of exhaustion marker expression, T1 was also characterized by tissue-resident memory T (TRM) cell feature for high expression of TRM marker genes, such as CD69, ITGAE (CD103) and ITGA ${ }^{32}$ (Fig. 2c).

The same method was applied to tumor-infiltrating Tregs and a total of 343 genes uniquely expressed by Tregs were identified (Supplementary Table 5). The TNBC Treg-specific genes largely overlapped with those identified in previous studies in liver cancer, melanoma and breast cancer ${ }^{31,33}$ (Fig. 2f).

\section{Pseudotime State Transition of T cells and a "Preexhaustion" T Cell Subset}

Pseudotime analysis provides us a method to depict the T cell developmental trajectories that correspond to biological processes such as activation or exhaustion, as recently suggested ${ }^{31}$. To probe into the $T$ cell functional state transition in TNBC, we used the Monocle2 algorithm to order $\mathrm{CD} 8^{+}$and $\mathrm{CD} 4^{+} \mathrm{T}$ cells in pseudotime based on transcriptional similarities ${ }^{34}$. The $\mathrm{CD}^{+} \mathrm{T}$ cell trajectory began with cells of $\mathrm{CCR} 7^{+}$ clusters T2 and T4, which were separately located at two different branches, followed by cytotoxic clusters T3 and T5, and terminated with exhausted clusters T1 and T6 (Fig. 3a). The enrichment of exhausted $T$ cells at the late period of the pseudotime was in line with previous analysis ${ }^{31,32}$, indicating the $\mathrm{CD} 8^{+} \mathrm{T}$ cell developmental process through activation to exhaustion. Similarly, we analyzed the CD $4^{+}$ T cells' differentiation trajectory, in which Treg clusters T7 and T9 were located at the other end of the 
exhausted $\mathrm{CD} 4^{+}$Th cluster T8, demonstrating distinct functional states among these T cell subsets (Fig. 3b).

As shown by the trajectory analysis of $\mathrm{CD}^{+} \mathrm{T}$ cells, $\mathrm{T} 5$ appeared to be an intermediate functional state, locating between cytotoxic cluster (T3) and exhausted clusters (T1 and T6). Comparison of the expression patterns of T5 and T3 revealed a set of 259 genes with elevated expression in T5, including exhausted marker genes CTLA4, HAVCR2, and TIGIT (Fig. 3c). Survival analysis of the BC cohort from METABRIC revealed that patients expressing $T 5^{\text {high }} \mathrm{T} 1^{\text {low }}$ or $T 3^{\text {high }} \mathrm{T} 1^{\text {low }}$ signature were associated with better overall survival and breast cancer specific survival than patients expressing $\mathrm{T} 1{ }^{\text {high }} \mathrm{T} 5^{\text {low }}$ or $\mathrm{T} 1^{\text {high }} \mathrm{T} 3^{\text {low }}$ signature, respectively (Fig. 3d; supplementary figure 3 ). Also, patients expressing high T3 and low T5 signature showed comparable survival when compared with high T5 and low T3 signature patients (Fig. 3d; supplementary figure 3). This survival disparity suggested that although T5 showed an elevated expression of exhausted markers, its signature was associated with more favorable prognosis than the exhausted T1 signature, consistent with the description of a "pre-exhaustion" T cell state that have been suggested in lung cancer and liver cancer ${ }^{31,32}$. Genes involved in the cellular senescence pathway, such as KDM6B and ZFP36L2 (Supplementary Table 2), were highly expressed by cells of T5, indicating a possible mechanism that triggers $T$ cell exhaustion ${ }^{35}$.

\section{Clonal Enrichment of Exhausted T Cells and Tregs in TNBC Microenvironment}

TCR analysis provides another approach to gain insight into the various states of $T$ cells. The diversity of TCR sequences is pivotal for recognizing viral antigens or tumor-specific neoantigens presented by the major histocompatibility complex (MHC) on antigen-presenting cells (APCs). While the TCR repertoire is enormous due to the large amount of TCRs and random recombination, identical TCR sequences can indicate $T$ cell clonal expansion. Our single-cell RNA-seq data allowed us to track the lineage of each $T$ cell based on their full-length TCR $\alpha$ and $\beta$ sequences assembled by the TraCeR method ${ }^{36}$. Full-length TCRs with both $\alpha$ and $\beta$ chains were obtained for 3,315 T cells from 14 patients, among which 2,311 harbored unique TCRs and 1,004 harbored repeatedly used TCRs, implying clonal expansion. Patterns of clonal expansion were detected at varying degrees in different $T$ cell clusters. While only approximately $10 \% \mathrm{CD}^{+}$cells of $\mathrm{CCR} 7^{+}$clusters (T2 and T4) harbored clonal TCRs (those whose $\alpha$ and $\beta$ TCR pairs were shared by at least two cells), the percentage reached over $30 \%$ in exhausted CD $8^{+} \mathrm{T}$ cell clusters (T1 and T6) (Fig. 3e). For the CD4 $4^{+}$clusters of Treg and exhausted Th, $17.82 \%$ to $30.61 \%$ of clonal $\mathrm{CD} 4^{+} \mathrm{T}$ cells were observed (Fig. 3e). Thus, identifying clonal TCRs at single-cell level verified the previously suggested activation and exhaustion status of different T cell clusters in TNBC microenvironment.

\section{A CXCL8-Producing CD8 ${ }^{+} \mathrm{T}$ Cell Subset Associated with Survival}

CXCL8 production is mostly ascribed to myeloid and epithelial cells, and previous studies have shown that human umbilical cord blood CD4 $4^{+}$naive $T$ cells can express CXCL8, but CXCL8-producing $T$ cells become rare in adults ${ }^{37}$. Here we observed T2, a new subset of $\mathrm{CD} 8^{+} \mathrm{CXCL} 8^{+} \mathrm{T}$ cells, which was found in 
10 of 14 TNBC tumors and accounted for $16.00 \%$ of the T cell population (Figure $4 a$ ). We also performed immunofluorescence testing to validated the existence of $\mathrm{CXCL} 8^{+} \mathrm{CD} 8^{+} \mathrm{T}$ cells, and quantification analysis showed consistent percentage of this subset of cells with RNA-seq result (Figure 4b). The broad existence of $\mathrm{CXCL}^{+} \mathrm{T}$ cells highlighted the possibility that this $\mathrm{T}$ cell subset might have active and potentially significant functions in TNBC microenvironment. By analyzing the T cell function-associated gene expression in T2, we found this subset of cells expressed high levels of naïve markers CCR7 and SELL, and activation marker CD69, but low levels of differentiation markers, including CD57 and KLRG1 (Supplementary Fig. 4a). These CXCL8 ${ }^{+} T$ cells expressed low levels of CXCR1 or CXCR2, the receptor for CXCL8, but elevated levels of other chemokines including CCL3, CCL4 and CCL2, when compared with T4, the other $\mathrm{CCR}^{+} \mathrm{T}$ cell cluster (Supplementary Table 6 ). These chemokines might work corporately with CXCL8 in TNBC microenvironment.

The observation of $\mathrm{CD} 8^{+} \mathrm{CXCL} 8^{+} \mathrm{T}$ cells was further confirmed using data from other single cell studies of breast cancer, liver cancer and colorectal cancer ${ }^{17,31,38,39}$. By defining CXCL8 positive using the cut-off of $\log _{2} T P M>1$, we found $C D 8^{+} C X C L 8^{+} T$ cell in all these four datasets (Supplementary Fig. 4b). We then explored whether $\mathrm{CD} 8^{+} \mathrm{CXCL} 8^{+} \mathrm{T}$ cells revealed in other single cell studies shared common characteristics with ours. We compared the high expressed genes ( $\left.\log _{2} \mathrm{FC}>1\right)$ between $\mathrm{CD} 8^{+} \mathrm{CXCL} 8^{+} \mathrm{T}$ cells observed in Zheng et al.'s study and that of our T2 cluster, which revealed 25 shared genes (Supplementary Fig. 4c). Among the 25 genes, nine were cytokines and chemokines, indicating the secretory function of this subset of $T$ cells.

Interestingly, a recent study revealed that $\mathrm{CD} 4^{+} \mathrm{CXCL} 8^{+} \mathrm{T}$ cells might support tumor growth and lymphoid metastasis via CXCL8-mediated neutrophil migration ${ }^{40}$. Consistently, survival analysis of an independent METABRIC cohort of breast cancer showed that patients with high expression of T2 signature genes and low T4 signature had significantly worse survival compared to those with high T4 and low T2 signature gene expression ( $P=0.0263$, Fig. 4c). To rule out the influence of CXCL8 produced by cells other than T cells, we further excluded CXCL8 from the signature genes used for the survival analysis, which consistently showed worse survival for patients with high T2 signature (Supplementary fig. 4d). These results suggest that $\mathrm{CXCL} 8^{+} \mathrm{CD} 8^{+} \mathrm{T}$ cells may associate with poor prognosis in breast cancer, illuminating a potential therapeutic target in TNBC.

To reveal the potential function of $\mathrm{CD} 8^{+} \mathrm{CXCL} 8^{+} \mathrm{T}$ cells in breast cancer, we performed DEG analysis between tumors showing high and low T2 signature using data from the METABRIC cohort. This analysis revealed 75 genes with elevated expression $\left(\log _{2} F C \geq 1\right)$ in the $T 2^{\text {high }}$ group (Supplementary Fig. $4 \mathrm{e}$; Supplementary Table 7). GO biological process enrichment analysis showed these highly expressed genes were generally enriched in the leukocytes (including granulocyte and myeloid leukocyte) chemotaxis and migration pathways (Fig. 4d; Supplementary Table 7). Moreover, this analysis also revealed that MAPK and ERK1/ERK2 cascades were activated in the $\mathrm{T} 2^{\text {high }}$ group (Supplementary Table 7). These results indicate that $\mathrm{CD} 8^{+} \mathrm{CXCL} 8^{+} T$ cells, a subset of chemokine-producing $T$ cells, might 
promote cancer progression through mediating leukocytes migration to tumor site and activating the MAPK/ERK pathways.

\section{Characterization of TAM subpopulations in TNBC}

For the categorization of the macrophages in TNBC, we first applied the classic macrophage polarization model. Based on the activation state, macrophages can be recognized as anti-tumoral classically activated (M1) cells and pro-tumoral alternatively activated (M2) cells ${ }^{41}$. We observed highly positive correlation of $\mathrm{M} 1$ and $\mathrm{M} 2$ signature gene expressions in all the macrophages we identified (Pearson correlation test, $\mathrm{R}=0.624, P<2.20 \times 10^{-16}$, Fig. 5a). Although this M1/M2 co-expressed state in single cell level raised challenge to the classic model of macrophage polarization showing exclusive discrete M1 and $\mathrm{M} 2$ activation state, along with recent report ${ }^{17,42}$, we were able to classify the macrophages into six clusters using Seruat based on their transcriptome features. Totally, we identified six macrophage clusters, each one of which had its uniquely expressed genes (Fig. 5b, Supplementary Table 8).

\section{Identification and Confirmation of TCR+ Macrophages in TNBC tumors}

Interestingly, we found a subset of macrophages specifically expressed T cell marker CD3 (Fig. 5c and Supplementary Fig. 5a), as well as the constant region of TCR $\alpha / \beta$ chains (Fig. $5 \mathrm{c}$ ), which suggested that these are macrophages bearing $\alpha / \beta$ TCR (or TCR ${ }^{+}$macrophages, the same hereinafter). Only paired productive TCRs can exert antigen recognition function. Thus, we explored whether these macrophages expressed paired productive TCRs by reconstructing the TCRs in these macrophages through the scRNAseq data. Totally, we identified $382 \mathrm{TCR}^{+}$macrophages with paired productive $\alpha$ and $\beta$ chains in 13 of the 14 patients, making up $14.28 \%$ of the total amount of macrophages, with the proportion ranged from $0.25 \%$ to $33.52 \%$ across patients (Supplementary Fig. $5 \mathrm{~b}$ ). The percentages of $\mathrm{TCR}^{+}$macrophages in MФ1 to MФ6 cluster ranged from $1.97 \%$ to $19.67 \%$ (Supplementary Fig. 5c). We considered these cells as macrophages rather than $\mathrm{T}$ cells for they clustered with other macrophages. In these cells, the expression levels of CD3 and TCR genes were between those for T cells and macrophages, while the macrophage markers had a similar expression level as in macrophages (Fig. $5 \mathrm{~d}$ ). In addition, through reference component analysis, we compared the transcriptome signature of $\mathrm{TCR}^{+}$macrophages with that of the reference bulk transcriptomes summarized by Li et al. ${ }^{25}$. We found that these cells were more similar to monocytes and macrophages, and were significantly different from T cells, B cells and NK cells (Supplementary Fig. 5e).

Next, we sought to validate the existence of $\mathrm{TCR}^{+}$macrophages. To verify the accuracy of TCR assembly and the expression of intact productive TCR $\alpha / \beta$ chains, we conducted Sanger sequencing validation in 10 selected single cells (including 5 macrophages and $5 \mathrm{~T}$ cells). The whole $\mathrm{V}$ region and $50 \mathrm{bp}$ of the $5^{\prime}$ $C$ region of $\alpha$ and $\beta$ chains were successfully amplified in 9 and 7 cells, respectively by 5 'race PCR in corresponding cDNA libraries. The Sanger results of all these amplicons were compared to the assembled TCR $\alpha / \beta$ chains of the corresponding cells as well as the VJ references from IMGT database. In V/J region, all amplified sequences reached more than $99.5 \%$ identity with IMGT references. And in the length 
of the amplicon, all assembled sequences reached more than $97 \%$ identity with corresponding sanger sequences (Supplementary Table 9).

Furthermore, we performed immunofluorescence assay on the tumor specimens of our study cohort to confirm the existence of these macrophage populations in TNBC tumor microenvironment. We observed the co-localization of CD68 and CD3/TCRa as well as CD14 and TCRa on the cell membrane in tumor infiltrated immune cells (Fig. 5e and Supplementary Fig. 5d, and Fig. 6), suggesting the presence of TCR ${ }^{+}$ macrophages. Additionally, we extended our validation by performing FACS analysis using tumor infiltrated immune cells isolated from fresh breast tumors of additional patients independent of our cohort. We found $26.58 \%$ and $25.96 \%$ of the tumor infiltrated CD68 ${ }^{+}$cells co-expressed CD3 and TCR, respectively (Fig. 5f). Taken together, these results reconfirmed that there is a subset of $\mathrm{TCR}^{+}$ macrophages in TNBC microenvironment.

\section{Possible TCR signaling pathways activation in $\mathrm{TCR}^{+}$Macrophages Revealed by Uniquely Expressed Gene Analysis}

We further investigated the differentially expressed genes of $\mathrm{TCR}^{+}$macrophages compared with $\mathrm{TCR}^{-}$ macrophages. Notably, significantly up-regulated genes included $L C K, L A T, F Y N$, and ICOS, which were necessary for TCR signaling in T lymphocytes, and GZMA, and GZMB, which were involved in the cytotoxic effect (Fig. 6a, b and Supplementary Table 10). Gene set enrichment analysis showed that the up-regulated genes in the TCR macrophages $\left(\log _{2} \mathrm{FC} \geq 1\right)$ were enriched in TCR signaling pathway, MAPK pathway, JAK-STAT signaling pathway, and nature killer cell mediate cytotoxicity pathway (Fig. $6 \mathrm{c})$. These findings illuminated that there might exist activated TCR signaling in these $\mathrm{TCR}^{+}$ macrophages. To verify the hypothesis, we examined the phosphorylation state of ZAP-70, a main switch controlling the activation of TCR signaling pathways ${ }^{43}$. We showed that phosphorylated ZAP-70 in TCR ${ }^{+}$ macrophages were observed in tumor specimens from multiple TNBC patients (Fig. 6d). We believe these findings raised a very interesting topic that worth future exploration-whether these $\mathrm{TCR}^{+}$macrophages can exhibit functions related to TCR signaling transduction.

\section{Discussion}

Despite major advances in cancer immunotherapy, our ability to understand mechanisms of action or predict efficacy is confounded by the heterogeneous composition of immune cells within tumors. The deep transcriptome data including the complete TCR information for 9,683 individual immune cells provided a comprehensive understanding and multi-dimensional characterization of TNBC infiltrated immune cells. The high quantity and quality of single-cell data allowed us to identify 22 immune cell clusters, including newly reported immune cell subsets, such as $\mathrm{CD} 8^{+} \mathrm{CXCL} 8^{+} \mathrm{T}$ cells and $\mathrm{TCR}^{+}$ macrophages. Full-length TCR-based analysis enabled us to depict cell clonal expansion patterns and cell lineages. In addition, the sequencing depth in our study assured reliable profiling of cytokines and transcription factors in each cell, allowing a detailed characterization of various immune cell subsets. 
Single-cell RNA sequencing or mass cytometry studies of breast tumors and other solid tumors have demonstrated the diversity of tumor-associated immune cell populations, which is supported by our observations in TNBC 17, 45 . In this study, we focused our interest on TNBC because it is a breast cancer subtype associated with rapid progression and poor survival once metastasized, although initial responses to chemotherapy and targeted agents sometimes happen. Immune checkpoint blockade therapy has shed light on TNBC treatment by slowing down disease progression and prolonging survival in selected patients ${ }^{21}$. A comprehensive knowledge of the TNBC immune ecosystem is pivotal for designing strategies to improve response to immunotherapy in this disease.

The level of tumor-infiltrated T cells and their characteristics are reported to be associated with outcomes of several cancers 9, 10,46. In our study, we identified T cells with different functional states and gene expression profiles. Savas et al. reported a group of $C D 8^{+} T_{R M}$ cells in TNBC microenvironment, characterizing as high levels of

immune checkpoint molecules expression ${ }^{39}$. Similar T cell subset (T1) was also observed in our analysis of a larger TNBC cohort, further highlighting its potential role in TNBC immune modulation or as a target for immunotherapy.

Pseudotime analysis demonstrated the $T$ cell functional state transition from activation to exhaustion along the developmental trajectory. We found the cluster T5, possibly representing cells in a transitional state from effector to exhaustion, indicating a "pre-exhaustion" state of T cells. The "pre-exhaustion" state of T cells has also been suggested in previous studies of NSCLC and HCC tumors ${ }^{31,32}$. Compared to effector T cells, "pre-exhaustion" T cells were characterized by lower expression of cytotoxic markers and elevated expression of exhaustion markers. Further, we found the "pre-exhaustion" signature correlated with better survival in a patient cohort when compared with the signature of exhausted T cell clusters. Thus, identifying these "pre-exhaustion" state T cells and finding ways to reverse these cells to an effector-like state or prevent them from progression into exhaustion could be a potential strategy of immunotherapy.

We report here a novel $\mathrm{T}$ cell subset of $\mathrm{CD} 8^{+} \mathrm{CXCL} 8^{+}$naïve $\mathrm{T}$ cells (T2), whose signature was linked to unfavorable prognosis. CXCL8-producing $\mathrm{CD}^{+}$naïve T cells exist in human peripheral blood, mainly in infants ${ }^{37}$. Crespo et al. found CXCL8-producing naive $\mathrm{CD} 4^{+} \mathrm{T}$ cells could mediate neutrophil migration in vitro and promotes primary ovarian cancer growth in animal model ${ }^{40}$. Consistently, we found the signature of $\mathrm{CD} 8^{+} \mathrm{CXCL} 8^{+} \mathrm{T}$ cell cluster is predictive of poor survival in a METABRIC patient cohort. Further DEG and pathway enrichment analysis indicated that tumors showing high T2 signature might progress through mediating leukocytes migration and activating the MAPK/ERK pathways. Taken together, the enrichment of $\mathrm{CXCL} 8^{+} \mathrm{CD} 8^{+} \mathrm{T}$ cells in the TME might contribute to the rapid progression and metastasis of TNBC. 
For macrophages, we showed a highly positive correlation between the M1 and M2 signature gene expressions at single cell level. These findings, raising challenges to the customary model of macrophage polarization that M1 and M2 activation states were regarded as mutually exclusive, supported the result of a recent single cell atlas study on breast cancer microenvironment reported by Azizi et al ${ }^{17,47}$. The coexistence and positive correlation of M1/M2 states highlighted the complexity of macrophage functionalities, helping to understand the role of TAM in modulating tumor microenvironment and design macrophage targeted therapies.

Notably, we found an interesting population of CD3 and TCR positive macrophages in this study. Previously, Alexander W. Beham and colleagues conducted a serial of convincing experiments to verify the presence of a T cell receptor (TCR) a $\beta$ based recombinational immune receptor in subpopulations of human and mouse monocytes and macrophages, by using flow cytometry, laser scanning cytometry (LSC), MALDI-TOF mass spectrometry, TCR $\beta$ gene locus recombination assay, immunofluorescence, RTPCR and TCR CDR3 length spectratyping analysis, western blot and immunoelectron microscopy ${ }^{48}$. Meanwhile, the authors also revealed that infection of macrophages from healthy individuals with mycobacteria triggered formation of clusters that express restricted TCR V $\beta$ repertoires ${ }^{48}$. Additionally, several studies have also observed the existence of the $\mathrm{TCR}^{+}$macrophages/monocytes and verified by different approaches in human peripheral blood, tumor microenvironment, artery, spleen and the bone marrow $49,50,51,52,53,54$. All these studies demonstrated the existence of macrophages/monocytes with TCRaß or TCR $\delta y$ in health and diseased human or murine tissue. Here, we for the first time observed this macrophage subset in breast cancer microenvironment, and investigated it at single-cell level. We found that $\mathrm{TCR}^{+}$macrophages might widely exist in TNBC tumors, illuminating that this long-neglected macrophage group might be an important component in tumor microenvironment.

Also, we suggested that there might be activation of TCR signaling pathway in tumor infiltrated $\mathrm{TCR}^{+}$ macrophages. These observations raised an attractive hypothesis that $\mathrm{TCR}^{+}$macrophages in tumor microenvironment might exert part of $\mathrm{T}$ cell-like functions. We assume that there may be two potential mechanisms that macrophages exhibit functional TCRs signaling. One possibility is that a proportion of macrophages express all the necessary recombination machinery to produce functional TCRs. On the other hand, given that macrophages eat cells as a part of their professional phagocytic functions ${ }^{55}$ and may also fuse with other cells 56,57 , it is reasonable to propose that a subset of macrophages in tumor microenvironment gain functions by using T cells' TCR mRNAs or proteins through eating or fusing with T cells. It is worthwhile to elucidate the underlying mechanisms in the future investigations. Additionally, whether $\mathrm{TCR}^{+}$macrophages work as a friend or foe with tumor cells in cancer progression deserves indepth exploration.

\section{Conclusion}

This study highlights the diverse phenotypes of immune cell populations in TNBC microenvironment. Single-cell sequencing data along with TCR information provide us a powerful tool to gain deep insights 
into the clustering, dynamics, developmental trajectory, and unique signatures of immune cell populations, enabling the discovery of novel immune cell subsets and potential molecular targets for immunotherapy. This immune cell atlas should be a valuable resource for future endeavor to identify clinically relevant cell signatures and predictive markers for precision immunotherapy.

\section{Methods}

Isolation of immune cells in human mammary carcinoma. Biopsies with a volume of $0.03-0.3 \mathrm{~cm}^{3}$ were mechanically disaggregated to $2 \mathrm{~mm}^{3}$ fragments followed by gentle 2 hours enzymatic dissociation with

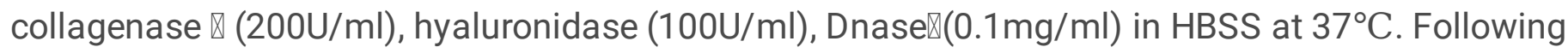
digestion, the cells were washed three times in HBSS. Immune cells were purified by FACS isolation of CD45 positive cells using APC Mouse Anti-Human CD45 (BD Pharmingen, clone: HI30 (RUO), American) as described by the manufacturer.

Single-cell suspension preparation and RNA extraction. Single-cell suspension preparation was carried out by the protocol previous described ${ }^{58}$. RNAs from the single-cells were extracted by a RNeasy plus mini kit (Qiagen) according to the manufacturer's instructions.

Single-cell library construction and sequencing. CDNA synthesis and amplification was performed as previous described ${ }^{58}$. Amplified cDNA products were purified by $1 \times$ Agencourt AMPure XP beads (Beckman Coulter). A total of $2 \mathrm{ng}$ purified cDNA products from each single-cell were used as the starting amount for library preparation. For the MIRALCS method, amplified cDNA was extracted by an automatic extractor from the chip to 96 -well plate and diluted from $200 \mathrm{nl}$ to $5 \mu$. And $3 \mu \mathrm{l} \mathrm{cDNA} \mathrm{products} \mathrm{without}$ purification were directly used for library construction. The libraries were prepared by TruePrep ${ }^{\text {TM }}$ Mini DNA Sample Prep Kit (Vazyme Biotech) according to the instruction manual and each sample was labelled with a barcode. All of the single-cells were sequenced on BGISEQ500 sequencing platform in a single-end mode.

Single-cell RNA-seq data processing. The single cell RNA-seq data was produced by BGISEQ-500 in SE100 single-end mode. First, we used Cutadapt ${ }^{59}$ to filter reads with adaptors $\Downarrow$ reads with more than five Ns and reads with low quality. Second, we used Bowtie setting parameters "-q --phred33 -sensitive --dpad 0 -gbar 99999999 --mp 1,1 -np 1 -score-min L,0, -0.1" to map the cleaned reads to the Ensembl hg38 human transcriptome. Then, we used RSEM (RNA-Seq by Expectation Maximization) algorithm to count the number of uniquely mapped reads pair for each gene and then calculate the TPM value for each gene. A readscount matrix and a TPM matrix was made for downstream analysis. We defined genes with TPM $>1$ as detected gene and the detected gene number is used for quality control. For each single cell, we calculated the average expression level $\left(\log _{2}(T P M+1)\right)$ of 96 curated housekeeping genes (Supplementary Table 4). To filter out cells with low quality, we set following standard to define high quality cells: 1).Mapping rates $\geq 50 \% ; 2$ ). Fraction of ERCC reads in mapped reads $<50 \% ; 3$ ). Mapped reads $\geq 2 \mathrm{M}$ (excluded ERCC reads); 4 ). Detected gene number $\geq 2500 ; 5)$. Housekeeping genes expression level (average $\log _{2}(T P M+1) \geq 4 ; 6$ ). Proportions of mitochondrial gene expression value (TPM value) < 
10\%. Cells defined as high quality were used for downstream analysis. 9683 cells passed the filtering and were used for downstream analysis.

Unsupervised clustering. We first normalized the TPM value as $\log _{2}(T P M+1)$. To remove the potential variation caused by individual differences, we further centered the log normalized TPM matrix patient by patient. If no explicitly stated, "normalized TPM" in this study refers to the log normalized and centered TPM value. We used Seurat2.0 to perform unsupervised clustering on the single cells using the normalized TPM matrix as input ${ }^{24}$. We kept genes annotated as "protein coding" for downstream analysis. Next, we considered genes with top 3000 highest standard deviation as highly variable genes excluded genes with average TPM $<1$ (not normalized囚across all cells to avoid unexpected noise. To regress out the variances caused by cycling cells from our data, we performed"Cell-Cycle Scoring and Regression"recommended by the tutorial of Seurat following the reported protocol ${ }^{60}$. In this step, single cells were scored basing on the scoring strategy described by Tirosh et al ${ }^{33}$ using a canonical gene list (Supplementary Table 5) and the relationship between gene expression and the cell cycle score was modeled to create a corrected expression matrix used for downstream dimensional reduction. After regressing out the cell cycle affect, we performed PCA on the corrected expression matrix using highly variable genes described above. Following the results of PCA, we selected the first 20 PCs for downstream analysis. Finally, we used the Seurat function "FindClusters" with the "resolution" parameter set to 1.5 to cluster the single cells. As a result, we obtained 22 cell clusters and visualized them by t-SNE.

Cell type annotation. To annotate our clusters to specific immune cell types (T cells, B cells, Macrophages, DCs, NKs), we selected some classic markers of these main immune cell types (Supplementary Table 6) and plotted their expression level on the t-SNE graph. Moreover, we analyzed differentially expressed genes (DEGs) among clusters to obtain the cluster specific genes of each cluster by $\mathrm{R}$ package limma ${ }^{61}$ using voom approach. The classic markers with adjusted $p$ value (BenjaminiHochberg multiple testing correction) $<0.01$, log2FC $\geq 3$ and expressed by $>50 \%$ of the cells $($ TPM $>1)$ in that cluster were used to define cell type (Supplementary Table 6). Furthermore, we compared cluster mean expression $\left(\log _{2}(T P M+1)\right)$ to sorted bulk transcriptome profile using $\mathrm{RCA}^{25}$. The results of marker annotating, DEG and RCA results were considered together to determine the cell type of our clusters. To further validate the clustering and cell type annotation results and regress out doublets and potential noise, we performed RCA on each single cell to annotate each single cell to a specific immune cell type and filtered out cells which were assigned to a different cell type with their cluster. We next identified $\mathrm{CD}^{+}$and $\mathrm{CD}^{+}$cells based on the expression of $C D 8 A, C D 8 B$ and $C D 4$ in the T cell population. Only cells with the average TPM of $C D 3 D, C D 3 E$ and $C D 3 G$ larger than 10 were kept for this analysis. Based on the average TPM of $C D 8 A$ and $C D 8 B$, one cell was considered as CD8 positive or negative if the value was larger than 7 or less than 1, respectively. Based on the TPM of CD4, one cell was considered as CD4 positive or negative if the value was larger than 7 or less than 1, respectively. As a result, cells were classified as $\mathrm{CD}^{+} \mathrm{CD}^{+}$(double positive, DP), $\mathrm{CD}^{-} \mathrm{CD} 8^{-}$(double negative, DN), $\mathrm{CD}^{-} \mathrm{CD}^{+}\left(\mathrm{CD} 8^{+} \mathrm{T}\right)$, $\mathrm{CD}^{+}{ }^{+} \mathrm{CD} 8^{-}\left(\mathrm{CD}^{+} \mathrm{T}\right)$ and other cells that cannot be clearly identified. Furthermore, $\mathrm{T} 1, \mathrm{~T} 2, \mathrm{~T} 3, \mathrm{~T} 4, \mathrm{~T} 5, \mathrm{~T} 6$ 
were defined as $\mathrm{CD} 8^{+} \mathrm{T}$ cluster while $\mathrm{T} 7, \mathrm{~T} 8, \mathrm{~T} 9$ as $\mathrm{CD} 4^{+} \mathrm{T}$ cluster according to the cell content of these $\mathrm{T}$ cell clusters.

Cluster marker analysis. In order to define the subtype of T cells and Macrophages, we analyzed the DEGs among $T$ cell clusters and Macrophage clusters separately by the following step $\otimes 1$ ). DEGs among clusters were analyzed by R package limma using voom approach. Genes with Benjamini-Hochbergadjusted F-test $P$-value $<0.01$ and $\log _{2} \mathrm{FC} \geq 1$ were kept. 2). The area under the curve value (AUC) was calculated for each gene to measure its ability to discriminate one cluster from the remaining clusters: each gene's log normalized TPM value was treated as a predictor, and cells inside and outside of the cluster were treated as positive and negative instances, respectively. Genes with AUC $\geq 0.5$ were kept. 3). The identified DEGs were then categorized to the cluster showing the highest $F C$ and with at least $50 \%$ of cells expressing this gene (TPM>0, Table S4).

CD45 isoform analysis. To reveal the composition of naïve and memory $T$ cell in each cluster, we analyzed the expression distribution of different CD45 isoforms. First, we utilized reads spanning the exons to represent the expression of CD 45 isoforms, for example, reads spanning the exon 3 and 7 (junction37) representing the expression of CD45RO. And then, we defined the normalized expression of junctions in each cell as $\mathrm{CPM}=\mathrm{Xi} / \mathrm{N} * 106$, where $\mathrm{Xi}$ represent the reads number of junctions, $\mathrm{N}$ denote the uniquely mapped reads number of each cells. Secondly, we removed 757 cells with the mean value and standard error of CPM of all junctions less than 1 and 0.2 respectively, because these cells could not reflect the real splicing structure of CD45. At last, a cell would be defined CD45RO ${ }^{+}$cell when the expression of $\mathrm{CD} 45 \mathrm{RO}^{+}$satisfy a threshold of $\log _{2}(\mathrm{CPM}+1)>1$.

DEG Analysis for Exhausted and Non-exhausted CD8+ T Cells. We defined cells in T1 as exhausted CD8+ T cells because there are several classic exhausted T cell markers such as HAVCR2, IDO1 and LAG3 significantly express in these clusters (adjusted $p$ value $<0.01$, fold change $>=4$ ). We defined cells in other CD8+ Tcell clusters as non-exhausted T cells. DEGs analysis between exhausted and nonexhausted CD8+ T cells was carried out using the voom method by limma described above with thresholds for adjusted $P$-value $<0.01$ and fold change $>=4$.

DEG Analysis for Treg and Other CD4+ T cells. Cells of T7 and T9 were defined as Treg while T8 as Tconv. We performed DEGs analysis using limma by voom approach described above between Treg and Tconv to get the Treg-specific genes with different threshold (adjusted $P$-value $<0.01$ and fold change $>=2$ ).

Cell developmental trajectory. We used the Monocle $2^{34}$ to order cells in $\mathrm{CD} 8^{+} / \mathrm{CD} 4^{+} \mathrm{T}$ cell clusters in pseudo time. We first used the "relative2abs" function in Monocle to convert TPM into normalized mRNA counts and created an object with parameter "expressionFamily = negbinomial.size" following the Monocle2 tutorial. We used "differentialGeneTest" function to derive DEG from clusters, keeping the genes with $Q$-value $<0.01$. After all, genes with mean expression $\geq 0.1$ (normalized mRNA read counts by "relative2abs" function) in the kept DEGs were used to order the cells in pseudo time. 
DEG analysis for exhausted and non-exhausted $\mathrm{CD}^{+} \mathrm{T}$ cells. We defined cells in $\mathrm{T} 1$ and $\mathrm{T} 6$ as exhausted $\mathrm{CD} 8^{+} \mathrm{T}$ cells and cells in other $\mathrm{CD} 8^{+} \mathrm{T}$ cell clusters as non-exhausted $\mathrm{CD} 8^{+} \mathrm{T}$ cells. DEGs analysis between exhausted and non-exhausted $\mathrm{CD} 8^{+} \mathrm{T}$ cells was carried out using the voom method by limma described above with thresholds for adjusted $P$-value $<0.01$ and log2FC $\geq 2$ (Table S5).

Survival analysis. The METABRIC ${ }^{63}$ dataset were used to evaluate the prognostic effect of signature gene sets derived from cluster. The gene expression and survival data of the METABRIC dataset were accessed using CBioPortal ${ }^{64}$. For each signature gene set, we calculated a "gene signature score" for each patient using fold-change values for each gene in the signature (Supplementary Table 1) to weight each gene in calculating the average. Then, patients were grouped into high and low expression groups by the median value of the "gene signature score". To correct clinical covariates including age and histological grade, we performed Multivariable analyses using Cox proportional hazards survival models and get the Hazard Ratio (HR) and adjusted $P$-value.

DEGs between patients grouped by the gene signature score of T2. As described in the "Survival analysis", patients were grouped into high and low expression groups by the median value of the "gene signature score" of T2. DEGs analysis between T2 ${ }^{\text {high }}(n=952)$ and T2 ${ }^{\text {low }}(n=952)$ patients was carried out following the best practice for microarray data using limma.

TCR Analysis. We used TraCeR ${ }^{34}$ method to assemble the TCR sequences for each single cell. Tracer can identify the rearranged TCR chains and use kallisto to calculate their TPM values. For every single cell, we rearranged its productive TCR chains by their TPM values. For example, if two TCRa chain were assembled in one single cell and they were both productive, the chain with higher TPM will be defined as TCRa1 while the chain with lower TPM as TCRa2.Non-productive TCR chains were excluded. The same rearrangement was deployed on TCR $\beta$. We kept cells with at least one pair of productive TCRa and TCR $\beta$ chain for subsequent analysis. We used a strict standard to define TCR clones: cells with the same TCRa1 and TCR $\beta 1$ were considered to be one TCR clone while the expanded clones were defined as clones with at least two cells sharing the same TCRa1 and TCR 1 in a given cell population.

When analyzing the sharing TCR within $\mathrm{CD} 8^{+} \mathrm{T}$ cells / $\mathrm{CD} 4^{+} \mathrm{T}$ cells or between $\mathrm{CD} 8^{+} / \mathrm{CD} 4^{+} \mathrm{T}$ cells and Macrophages, only cells defined as $\mathrm{CD} 4^{-} \mathrm{CD} 8^{+} \mathrm{T}$ cells or $\mathrm{CD} 4^{+} \mathrm{CD} 8^{-} \mathrm{T}$ cells according to $\mathrm{CD} 4 / \mathrm{CD} 8$ expression (see the cell type annotation section) were included in the analysis

TCR sequence sanger validation. Single cells' mRNA was reverse transcripted into cDNA during the RNAseq already. The CDNA was amplificated with TCR primer by three step nest-PCR. First PCR needs $2 \times$ KAPA Ready mix $12.5 \mu \mathrm{M}, 10 \mathrm{uM}$ IS PCR primer $0.5 \mu \mathrm{M}$, 10uM CA-out (5'-GCAGACAGACTTGTCACTGG-3') $1 \mu \mathrm{M}, 10 \mathrm{uM}$ CB-out (5'-TGGTCGGGGAAGAAGCCTGTG-3') $1 \mu \mathrm{M}$, RT mix $10 \mu \mathrm{M}$, and the amplification steps were $98^{\circ} \mathrm{C} 3 \mathrm{~min} ; 98^{\circ} \mathrm{C} 15 \mathrm{~s}, 55^{\circ} \mathrm{C} 20 \mathrm{~s}, 72^{\circ} \mathrm{C} 2 \mathrm{~min} \times 25$ cycles; $72{ }^{\circ} \mathrm{C} 5$ min, $4{ }^{\circ} \mathrm{C}$ hold. The second PCR has $2 \times$ KAPA Ready mix $12.5 \mu \mathrm{M}, 10 \mathrm{uM}$ IS PCR primer $0.5 \mu \mathrm{M}$, 10uM CA-in-N (5'-

AGTCTCTCAGCTGGTACACG-3') $1 \mu \mathrm{M}$, 10uM CB-in-N (5'-GCAGACAGACTTGTCACTGG-3') $1 \mu \mathrm{M}$, the first PCR RT mix $10 \mu \mathrm{M}$, and the amplification steps were $98^{\circ} \mathrm{C} 3 \mathrm{~min} ; 98^{\circ} \mathrm{C} 15 \mathrm{~s}, 60^{\circ} \mathrm{C} 20 \mathrm{~s}, 72{ }^{\circ} \mathrm{C} 2 \mathrm{~min} \times 25$ 
cycles; $72{ }^{\circ} \mathrm{C} 5 \mathrm{~min}, 4^{\circ} \mathrm{C}$ hold. The third PCR contains $2 \times$ KAPA Ready mix $12.5 \mu \mathrm{M}, 10 \mathrm{MM}$ IS PCR primer

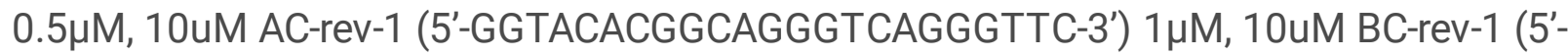

TTCTGATGGCTCAAACACAGCGA-3') $1 \mu \mathrm{M}$, the second PCR RT mix $10 \mu \mathrm{M}$, and the amplification steps were $98^{\circ} \mathrm{C} 3 \mathrm{~min} ; 98^{\circ} \mathrm{C} 15 \mathrm{~s}, 60^{\circ} \mathrm{C} 20 \mathrm{~s}, 72{ }^{\circ} \mathrm{C} 2 \mathrm{~min} \times 35$ cycles; $72{ }^{\circ} \mathrm{C} 5 \mathrm{~min}, 4^{\circ} \mathrm{C}$ hold. Then $1 \%$ agarose gel electrophoresis was used to recover the target DNA. Connected DNA fragment to pMDTM18-T vector (TaKaRa 6011) at $4{ }^{\circ} \mathrm{C}$ overnight. Transformed the product into Trans5a (Transgen, CD201), and plated the bacterial on ager medium with Ampicillin, X-Gal and ITPG at $37^{\circ} \mathrm{C}$ for $16 \mathrm{~h}$. Picked one white bacterial colony to $20 \mu \mathrm{lddH} 20$, then took $1 \mu \mathrm{l}$ to PCR with R primer (5'-CGCCAGGGTTTTCCCAGTCACGAC-3') $1 \mu \mathrm{l}$, and F primer $1 \mu \mathrm{l}$, dNTPs $4 \mu \mathrm{l}$, r-Taq $1 \mu \mathrm{l}, 10 \times$ Buffer $5 \mu \mathrm{l}$, ddH2O 37 $\mu$ l. The amplification steps were $98^{\circ} \mathrm{C}$ $5 \mathrm{~min} ; 95^{\circ} \mathrm{C} 30 \mathrm{~s}, 60^{\circ} \mathrm{C} 30 \mathrm{~s}, 72^{\circ} \mathrm{C} 40 \mathrm{~s} \times 35$ cycles; $72{ }^{\circ} \mathrm{C} 10 \mathrm{~min}, 4^{\circ} \mathrm{C}$ hold. Performed $1 \%$ agarose gel electrophoresis to recover the target DNA. The recovered DNA was sequenced by sanger sequencing.

TCR sanger sequencing result analysis. Sanger sequencing was performed and we obtained the TCR sequence of some of the single cells. We blast the sequence against the sequence assembled by Tracer method and the VDJ sequences in IMGT database to proof the result of Tracer (Supplementary Table 9).

$M 1$ and $M 2$ signature analysis. $M 1$ and $M 2$ signature genes were provided by Azizi.et al ${ }^{17}$. The normalized TPM (see the unsupervised clustering section) was used for analysis. We calculated the mean expression of M1/M2 signature for each cell and calculated the Pearson correlation of M1 and M2 signature.

Flow Cytometry. $5 \times 5 \times 5 \mathrm{~mm}$ tumor samples were resected during the operation and cut into pieces. Added the digestive fluid to the tubes and isolated the tissue into single cells. Dyed the cells with TCR $\alpha / \beta$ PE (555548, BD), CD68 FITC (333806, Biolegend), CD3 APC (17-0036-42, eBioscience), CD45RA PE-CY7 (560675, BD) , CD45RO PE (304206, Biolegend) , CD4 PE 300508, Biolegend) , CD8 FITC (557085, BD) antibodies, grouped the samples and analyzed with CytoFLEX FCM (Beckman). In order to exclude the effect of non-specific cell staining, IgG isotype control was used. Positive cells were distinguished based on isotype controls.

Immunofluorescence. The tissue sections were placed at room temperature for $1 \mathrm{~h}$ before dewaxing. Soaked the sections in dimethylbenzene for $10 \mathrm{~min}$ and change the dimethylbenzene for $10 \mathrm{~min}$ more. Immersed the sections in 100\% -95\%- 85\%-75\% ethanol for 5 min each, washed by PBS for $5 \mathrm{~min}$ twice. Bathed the sections in $0.01 \mathrm{M}$ sodium citrate buffer solution $(\mathrm{pH} 6.0)$ at $95^{\circ} \mathrm{C}$ for $15 \mathrm{~min}$, washed with PBS for $5 \mathrm{~min}$. Added $5 \%$ goat serum to the slides and sealed at room temperature for 30 minutes. Added CD3 (ZSGB-BIO, ZA-0503), CD68(ZSGB-BIO, ZM-0464), ZAP70(Abcam, ab76306), TCR a (Abcam, ab18861), CD14 (Abcam, ab182032), CD8 (Abcam, ab237709), CXCL8 (Abcam, ab18672), CD56 (Abcam, ab9018), primary antibody $(1: 200)$ overnight at $4^{\circ} \mathrm{C}$, washed with PBS for $5 \mathrm{~min}$ three times. The fluorescently labeled secondary antibody (1:100) Dylight 488 (Abbkine, A23220) and Dylight 549 (Abbkine, A23310) was incubated for $1 \mathrm{~h}$ at room temperature in the dark. Bathed in PBS for $5 \mathrm{~min}$ twice, dropped DAPI (Abcam, ab104139) and applied cover slip carefully. Sealed with nail polish and dried at room 


\section{Declarations}

\section{Acknowledgements}

The authors would like to thank professor Xi Wang, Jun Tang, Xiangsheng Xiao and Weidong Wei for contributing TNBC samples. We thanked Fei Wang for his technical support in single cell TCR sequencing and validation. We thanked Dr. Song Gao, and Dr. Leo J Lee for their valuable advices for this paper.

\section{Authors' contributions}

S.W., B.L., Q.Z., S.Q. and R.H. conceived the project. Y.L., L.Z., W.Z. and X.L. designed the experiments. Y.L., L.Z., X.L., K.Z., M.H., K.L. and W.Z. performed the experiments. Q.L., W.X., F.X, Q.Z. and K.L. collected clinical samples. S.Q., Z.Z., S.L analyzed the sequencing data. S.Q., R.H., S.W., B.L., Z.Z., Y.L., Z.L. W.Z. wrote the manuscript with all authors contributing to writing and providing feedback.

\section{Funding}

This project was supported by grants from Joint Fund of the National Natural Science Foundation of China and Natural Science Foundation of Guangdong Province (U1601224), Natural Science Foundation of Guangdong Province (2020A1515010105) and Shenzhen Municipal Government of China (No. 20170731162715261)

\section{Availability of data and materials}

The Single-cell RNA sequencing data in this study are available in the CNGB Nucleotide Sequence Archive (CNSA: https://db.cngb.org; accession number: CNP0000286).

\section{Ethics approval and consent to participate}

This study was approved by the Ethical Committee of Sun Yat-sen University Cancer Center and complied with all relevant ethical regulations. Written informed consent was provided by all patients. All experimental methods abided by the Helsinki Declaration.

\section{Consent for publication}

Not applicable.

\section{Competing interests}

The authors declare that they have no competing interests. 


\section{References}

1. Dehne, N., Mora, J., Namgaladze, D., Weigert, A. \& Brune, B. Cancer cell and macrophage cross-talk in the tumor microenvironment. Curr Opin Pharmaco/ 35, 12-19 (2017).

2. Kim, J. \& Bae, J.S. Tumor-Associated Macrophages and Neutrophils in Tumor Microenvironment. Mediators Inflamm 2016, 6058147 (2016).

3. Engblom, C., Pfirschke, C. \& Pittet, M.J. The role of myeloid cells in cancer therapies. Nat Rev Cancer 16, 447-462 (2016).

4. Pyonteck, S.M. et al. CSF-1R inhibition alters macrophage polarization and blocks glioma progression. Nature medicine 19, 1264-1272 (2013).

5. Ries, C.H. et al. Targeting tumor-associated macrophages with anti-CSF-1R antibody reveals a strategy for cancer therapy. Cancer cell 25, 846-859 (2014).

6. Havel, J.J., Chowell, D. \& Chan, T.A. The evolving landscape of biomarkers for checkpoint inhibitor immunotherapy. Nature reviews. Cancer 19, 133-150 (2019).

7. Galon, J. et al. Type, density, and location of immune cells within human colorectal tumors predict clinical outcome. Science 313, 1960-1964 (2006).

8. Vesely, M.D., Kershaw, M.H., Schreiber, R.D. \& Smyth, M.J. Natural innate and adaptive immunity to cancer. Annual review of immunology 29, 235-271 (2011).

9. Ovarian Tumor Tissue Analysis, C. et al. Dose-Response Association of CD8 + Tumor-Infiltrating Lymphocytes and Survival Time in High-Grade Serous Ovarian Cancer. JAMA Oncol 3, e173290 (2017).

10. Kansy, B.A. et al. PD-1 Status in CD8(+) T Cells Associates with Survival and Anti-PD-1 Therapeutic Outcomes in Head and Neck Cancer. Cancer Res 77, 6353-6364 (2017).

11. Speiser, D.E., Ho, P.C. \& Verdeil, G. Regulatory circuits of T cell function in cancer. Nat Rev Immunol 16, 599-611 (2016).

12. Wherry, E.J. \& Kurachi, M. Molecular and cellular insights into T cell exhaustion. Nat Rev Immuno/ 15, 486-499 (2015).

13. Kitamura, T., Qian, B.Z. \& Pollard, J.W. Immune cell promotion of metastasis. Nature reviews. Immunology 15, 73-86 (2015).

14. Chanmee, T., Ontong, P., Konno, K. \& Itano, N. Tumor-associated macrophages as major players in the tumor microenvironment. Cancers 6, 1670-1690 (2014).

15. Sica, A. et al. Macrophage polarization in tumour progression. Seminars in cancer biology 18, 349355 (2008).

16. Ginhoux, F., Schultze, J.L., Murray, P.J., Ochando, J. \& Biswas, S.K. New insights into the multidimensional concept of macrophage ontogeny, activation and function. Nature immunology 17, 34-40 (2016). 
17. Azizi, E. et al. Single-Cell Map of Diverse Immune Phenotypes in the Breast Tumor Microenvironment. Cell 174, 1293-1308 e1236 (2018).

18. Foulkes, W.D., Smith, I.E. \& Reis-Filho, J.S. Triple-negative breast cancer. N Engl J Med 363, 19381948 (2010).

19. Denkert, C., Liedtke, C., Tutt, A. \& von Minckwitz, G. Molecular alterations in triple-negative breast cancer-the road to new treatment strategies. Lancet 389, 2430-2442 (2017).

20. Jiang, Y.Z. et al. Genomic and Transcriptomic Landscape of Triple-Negative Breast Cancers: Subtypes and Treatment Strategies. Cancer Cell 35, 428-440 e425 (2019).

21. Schmid, P. et al. Atezolizumab and Nab-Paclitaxel in Advanced Triple-Negative Breast Cancer. The New England journal of medicine 379, 2108-2121 (2018).

22. Early Pembrolizumab Ups TNBC Responses. Cancer Discov 9, 1638 (2019).

23. Schmid, P. et al. Atezolizumab plus nab-paclitaxel as first-line treatment for unresectable, locally advanced or metastatic triple-negative breast cancer (IMpassion130): updated efficacy results from a randomised, double-blind, placebo-controlled, phase 3 trial. Lancet Oncol (2019).

24. Butler, A., Hoffman, P., Smibert, P., Papalexi, E. \& Satija, R. Integrating single-cell transcriptomic data across different conditions, technologies, and species. Nat Biotechnol 36, 411-420 (2018).

25. Li, H. et al. Reference component analysis of single-cell transcriptomes elucidates cellular heterogeneity in human colorectal tumors. Nat Genet 49, 708-718 (2017).

26. Collin, M., McGovern, N. \& Haniffa, M. Human dendritic cell subsets. Immunology 140, 22-30 (2013).

27. Pieper, K., Grimbacher, B. \& Eibel, H. B-cell biology and development. The Journal of allergy and clinical immunology 131, 959-971 (2013).

28. Förster, R., Davalos-Misslitz, A.C. \& Rot, A. CCR7 and its ligands: balancing immunity and tolerance. Nature Reviews Immunology 8, 362 (2008).

29. Chen, L. \& Flies, D.B. Molecular mechanisms of T cell co-stimulation and co-inhibition. Nat Rev Immunol 13, 227-242 (2013).

30. Grogg, K.L. et al. Expression of CXCL13, a chemokine highly upregulated in germinal center T-helper cells, distinguishes angioimmunoblastic T-cell lymphoma from peripheral T-cell lymphoma, unspecified. Mod Pathol 19, 1101-1107 (2006).

31. Zheng, C. et al. Landscape of Infiltrating T Cells in Liver Cancer Revealed by Single-Cell Sequencing. Cell 169, 1342-1356 e1316 (2017).

32. Guo, X. et al. Global characterization of T cells in non-small-cell lung cancer by single-cell sequencing. Nat Med 24, 978-985 (2018).

33. Tirosh, l. et al. Dissecting the multicellular ecosystem of metastatic melanoma by single-cell RNAsEq. Science 352, 189-196 (2016).

34. Trapnell, C. et al. The dynamics and regulators of cell fate decisions are revealed by pseudotemporal ordering of single cells. Nat Biotechnol 32, 381-386 (2014). 
35. Salminen, A., Kaarniranta, K., Hiltunen, M. \& Kauppinen, A. Histone demethylase Jumonji D3 (JMJD3/KDM6B) at the nexus of epigenetic regulation of inflammation and the aging process. $J \mathrm{Mol}$ Med (Berl) 92, 1035-1043 (2014).

36. Stubbington, M.J.T. et al. T cell fate and clonality inference from single-cell transcriptomes. Nat Methods 13, 329-332 (2016).

37. Gibbons, D. et al. Interleukin-8 (CXCL8) production is a signatory T cell effector function of human newborn infants. Nature medicine 20, 1206-1210 (2014).

38. Zhang, L. et al. Lineage tracking reveals dynamic relationships of T cells in colorectal cancer. Nature 564, 268-272 (2018).

39. Savas, P. et al. Single-cell profiling of breast cancer T cells reveals a tissue-resident memory subset associated with improved prognosis. Nat Med 24, 986-993 (2018).

40. Crespo, J. et al. Human Naive T Cells Express Functional CXCL8 and Promote Tumorigenesis. Journal of immunology 201, 814-820 (2018).

41. Mantovani, A. \& Locati, M. Tumor-associated macrophages as a paradigm of macrophage plasticity, diversity, and polarization: lessons and open questions. Arterioscler Thromb Vasc Bio/33, 14781483 (2013).

42. Muller, S. et al. Single-cell profiling of human gliomas reveals macrophage ontogeny as a basis for regional differences in macrophage activation in the tumor microenvironment. Genome Bio/ 18, 234 (2017).

43. Wang, H. et al. ZAP-70: an essential kinase in T-cell signaling. Cold Spring Harbor perspectives in biology 2, a002279 (2010).

44. Simoni, Y. et al. Bystander CD8 + T cells are abundant and phenotypically distinct in human tumour infiltrates. Nature 557, 575 (2018).

45. Wagner, J. et al. A Single-Cell Atlas of the Tumor and Immune Ecosystem of Human Breast Cancer. Cell 177, 1330-1345 e1318 (2019).

46. Wu, S.P. et al. Stromal PD-L1-Positive Regulatory T cells and PD-1-Positive CD8-Positive T cells Define the Response of Different Subsets of Non-Small Cell Lung Cancer to PD-1/PD-L1 Blockade Immunotherapy. Journal of thoracic oncology: official publication of the International Association for the Study of Lung Cancer 13, 521-532 (2018).

47. Cassetta, L. et al. Human Tumor-Associated Macrophage and Monocyte Transcriptional Landscapes Reveal Cancer-Specific Reprogramming, Biomarkers, and Therapeutic Targets. Cancer Cell 35, 588602 e510 (2019).

48. Beham, A.W. et al. A TNF-regulated recombinatorial macrophage immune receptor implicated in granuloma formation in tuberculosis. PLoS Pathog 7, e1002375 (2011).

49. Fuchs, T. et al. The neutrophil recombinatorial TCR-like immune receptor is expressed across the entire human life span but repertoire diversity declines in old age. Biochem Bioph Res Co 419, 309315 (2012). 
50. Korin, B. et al. High-dimensional, single-cell characterization of the brain's immune compartment. Nat Neurosci 20, 1300-1309 (2017).

51. Fuchs, T. et al. A combinatorial alphabeta T cell receptor expressed by macrophages in the tumor microenvironment. Immunobiology 222, 39-44 (2017).

52. Fuchs, T. et al. The macrophage-TCR alpha beta is a cholesterol-responsive combinatorial immune receptor and implicated in atherosclerosis. Biochem Bioph Res Co 456, 59-65 (2015).

53. Fuchs, T. et al. A second combinatorial immune receptor in monocytes/macrophages is based on the TCR gamma delta. Immunobiology 218, 960-968 (2013).

54. Chorazeczewski, J.K. et al. TCR beta Combinatorial Immunoreceptor Expression by Neutrophils Correlates with Parasite Burden and Enhanced Phagocytosis during a Plasmodium berghei ANKA Malaria Infection. Infect Immun 86 (2018).

55. Lemke, G. How macrophages deal with death. Nat Rev Immunol 19, 539-549 (2019).

56. Bracq, L. et al. T Cell-Macrophage Fusion Triggers Multinucleated Giant Cell Formation for HIV-1 Spreading. J Viro/ 91 (2017).

57. Vignery, A. Macrophage fusion: are somatic and cancer cells possible partners? M S-Med Sci 21, 1070-1075 (2005).

58. Wu, L. et al. Full-length single-cell RNA-seq applied to a viral human cancer: applications to HPV expression and splicing analysis in HeLa S3 cells. GigaScience 4, 51-51 (2015).

59. Martin, M.J.E.J. Martin M.Cut adapt removes adapter sequences from high-throughput sequencing reads, EMBnet. EMBnet 17:10-12. 17 (2011).

60. Nestorowa, S. et al. A single cell resolution map of mouse haematopoietic stem and progenitor cell differentiation. (2016).

61. Ritchie, M.E. et al. limma powers differential expression analyses for RNA-sequencing and microarray studies. Nucleic Acids Research 43, e47-e47 (2015).

62. Huang, M. et al. SAVER: gene expression recovery for single-cell RNA sequencing. Nat Methods 15, 539-542 (2018).

63. Curtis, C. et al. The genomic and transcriptomic architecture of 2,000 breast tumours reveals novel subgroups. Nature 486, 346-352 (2012).

64. Gao, J. et al. Integrative analysis of complex cancer genomics and clinical profiles using the cBioPortal. Science signaling 6, pl1-pl1 (2013).

\section{Figures}




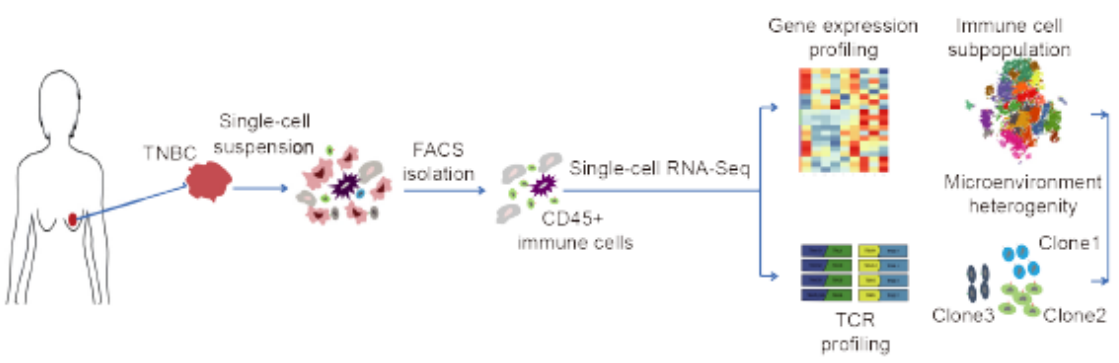

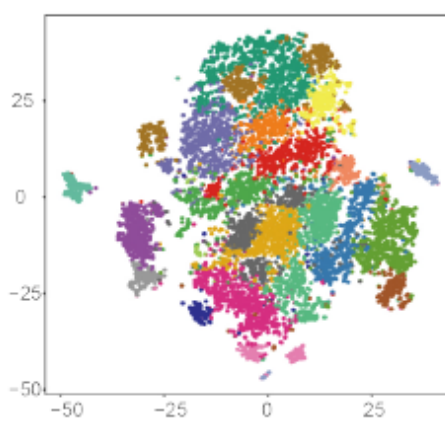
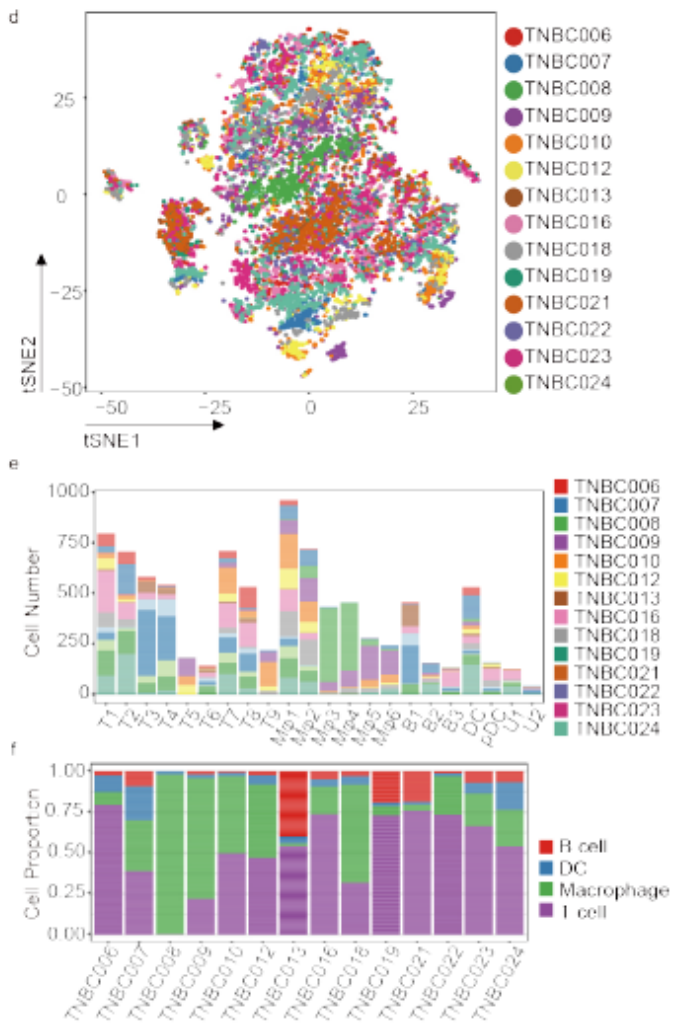
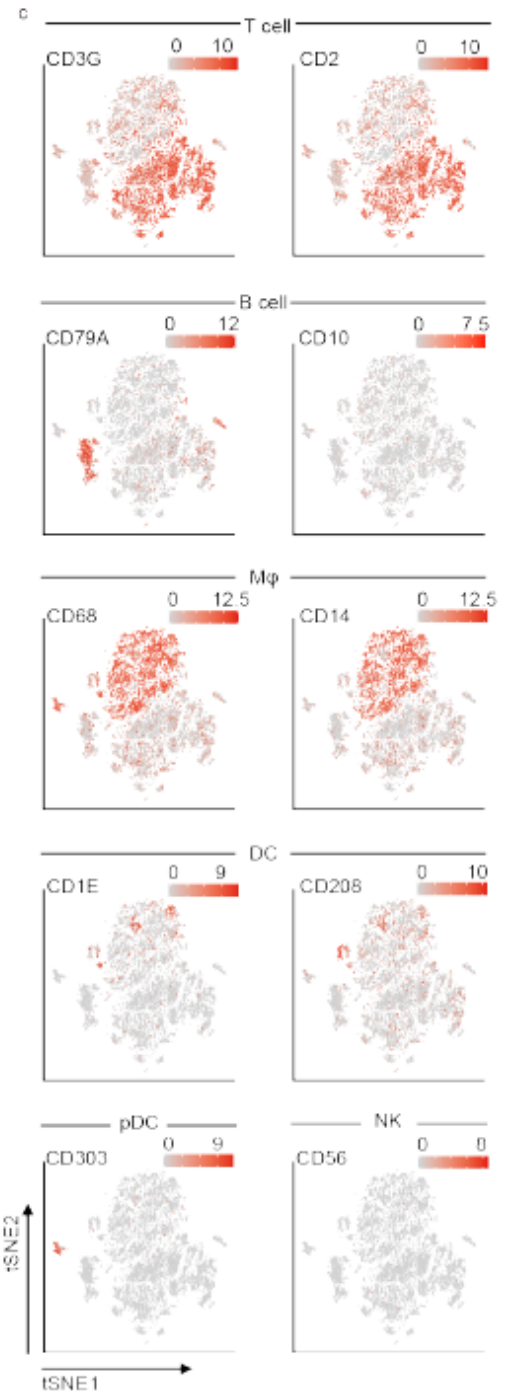

\section{Figure 1}

Program Design and Immune Landscape of TNBC. a, Flow chart of the experimental design. Single-cell RNA sequencing was applied to CD45+ cells derived from tumor tissue. The scRNA-seq data were then subjected to gene expression profiling and TCR profiling. b, Two-dimensional t-SNE plot of 9,683 CD45+ single cells from 14 TNBC patients. Each point represents one single cell囚colored according to cell cluster. c, Cells colored by normalized expression $(\log 2(T P M+1))$ of immune cell type classification markers on 
the t-SNE map. d, Similar to b, colored according to patient. e, Bar plot shows the fractions of cells from different patients in each cell cluster, colored by patient. $f$, Bar plot shows the cell type fractions in each patient's tumor-infiltrating immune cells, colored by cell type. T1-T9, T cell clusters. MФ1-MФ6, macrophage clusters. B1-B3, B cell clusters. DC and pDC, DC clusters. U1 and U2, undefined clusters.

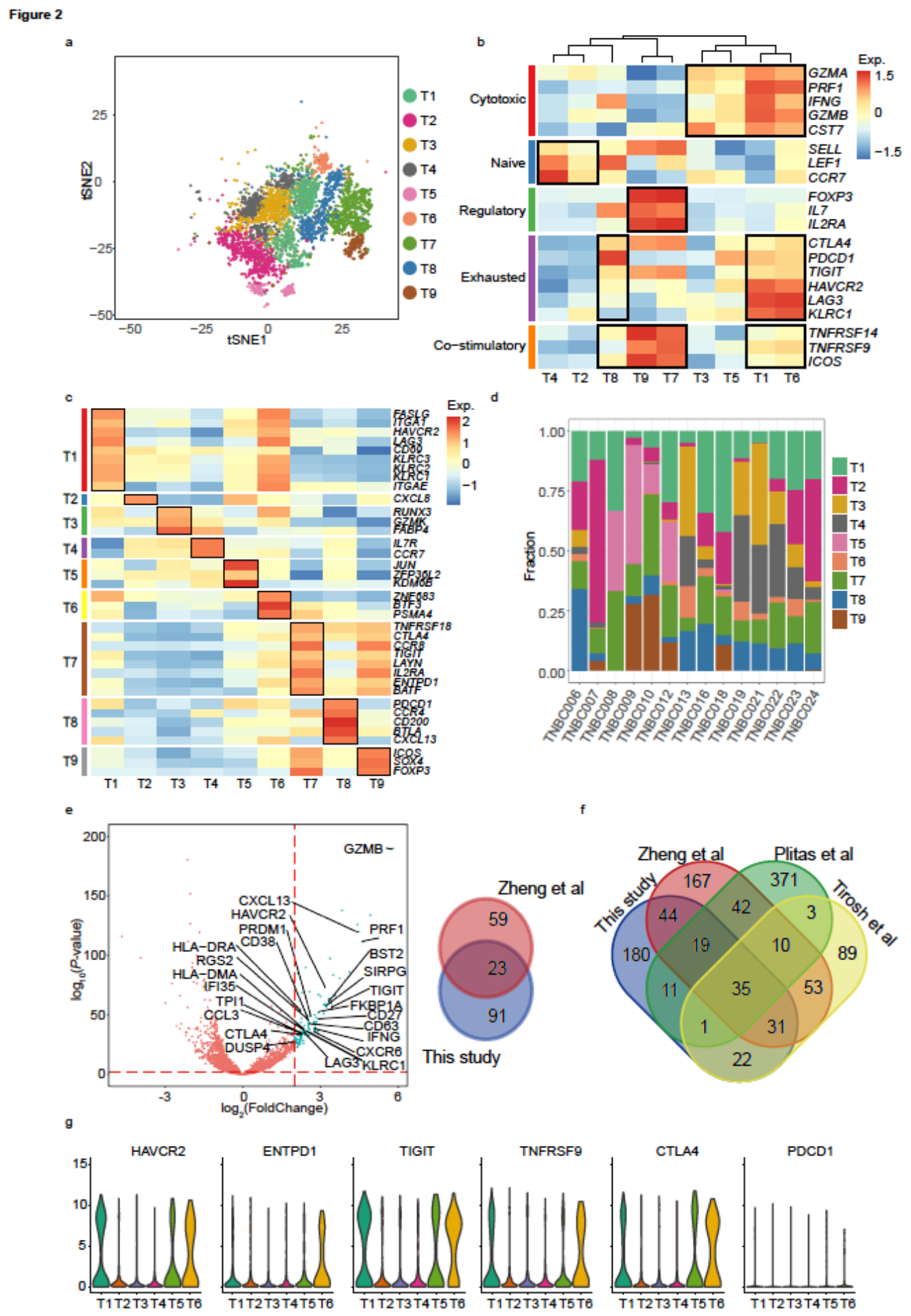

Figure 2 
Clustering and Functional Analysis of T Cells. a, Two-dimensional t-SNE plot of 9 identified T cell clusters. Each point represents one single cell, colored according to cell cluster. b, Z-score normalized mean expression $(\log 2(T P M+1))$ of selected T cell function-associated genes in each T cell cluster. Black boxes highlight the patterns defining known T cell subtypes. c, Z-score normalized mean expression $(\log 2(T P M+1))$ of most representative markers of each $T$ cell cluster. Black boxes highlight the markers for specific cluster. $d$, The fractions of nine T cell clusters within each patient. e, The volcano plot in the left panel shows differentially expressed genes in tumor-infiltrating exhausted T cells. Each blue dot represents a gene with $P$-value $\otimes 0.01$ and $\log 2 F C \geq 2$. The right panel shows the overlap of exhausted $T$ cell signature genes identified in this study with those from previous study by Zheng et al. (2017) ( $p<$ 2.2e-64), determined by hypergeometric test. Totally 23 genes overlap with previous HCC study by Zheng et al. (2017), which are marked with gene names. f, Venn graph showing the overlap of Treg signature genes identified in this study with those from previous studies by Zheng et al. (2017), Plitas et al. (2016), and Tirosh et al. (2016). g, The expression levels of exhausted markers in CD8+ T cell clusters. 

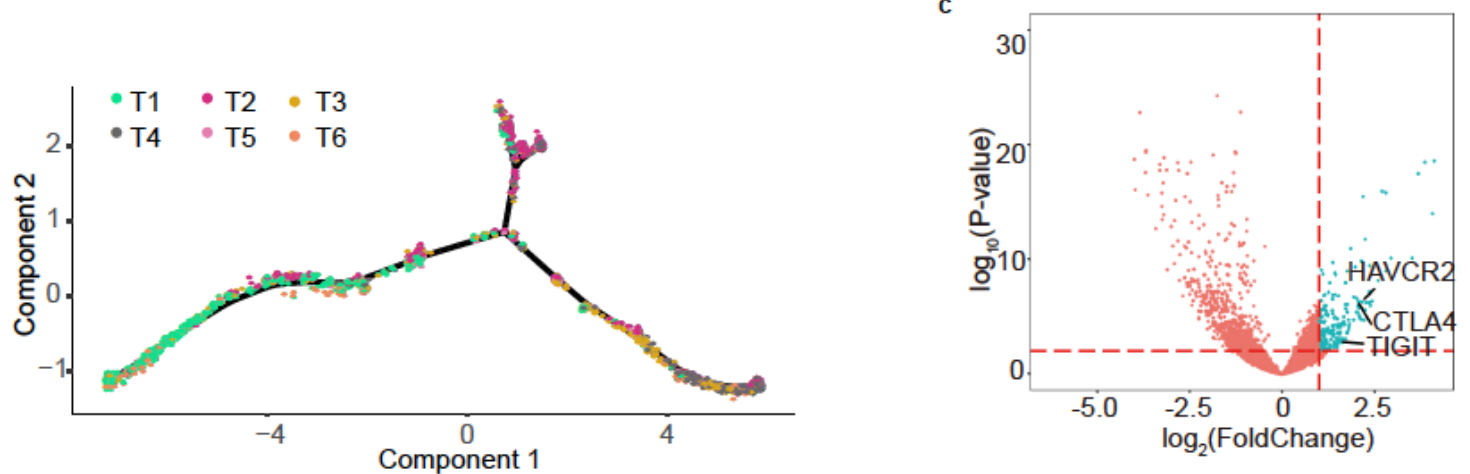

b

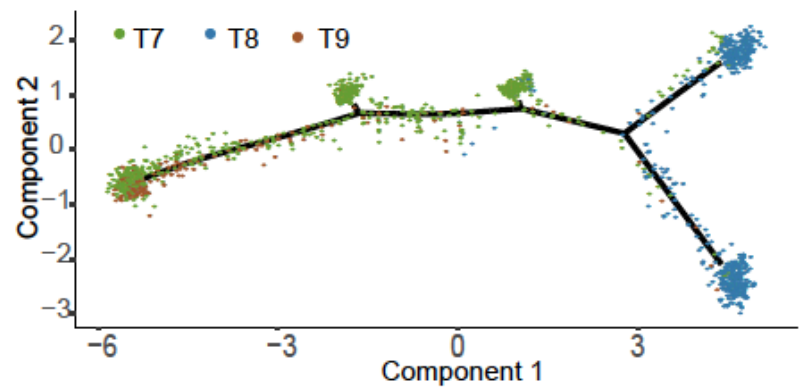

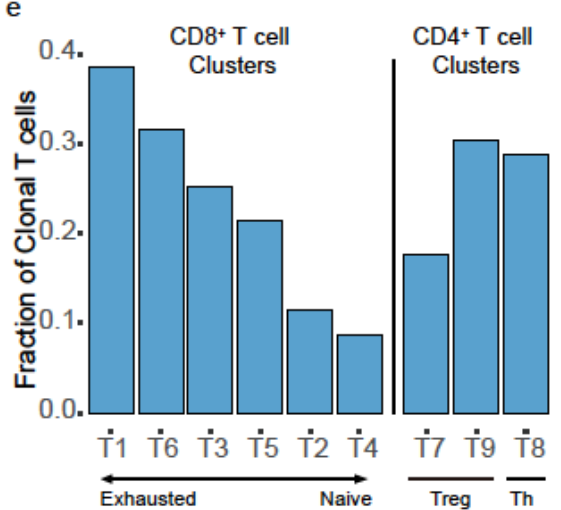

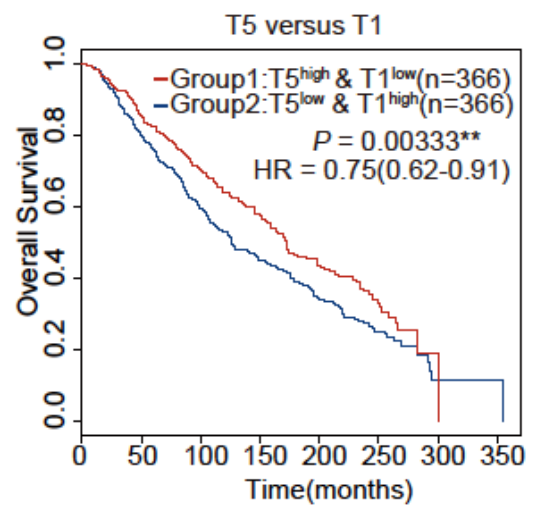

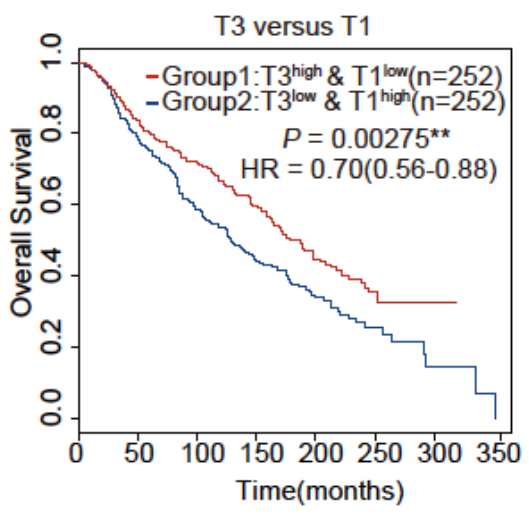

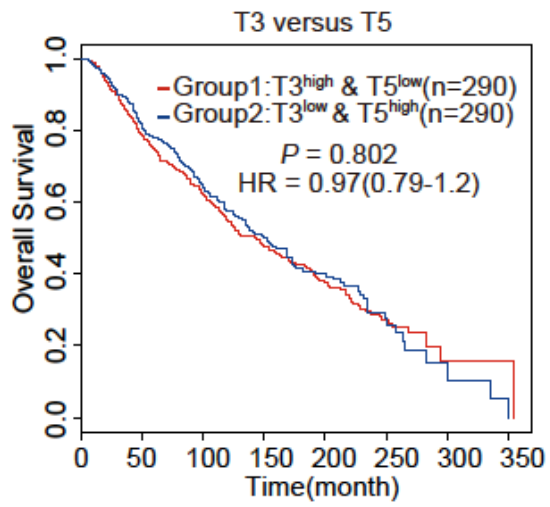

\section{Figure 3}

T Cell Trajectory Analysis Reveals a "Pre-exhaustion" T Cell Subset. a, The trajectory of CD8+ T cells shows CD8+ T cell state transition in a two-dimensional space defined by Monocle2. Each point represents one single cell, colored according to cell cluster. b, Similar plot as a for CD4+ T cell clusters. c, Differential genes between T3 and T5. Each blue dot represents a gene with P-value $₫ 0.01$ and log2FC $\geq$ 2. $d$, The Kaplan-Meier overall survival curves of METABRIC patients grouped by the gene signature score (see Method) of T5 and T1(left panel), T3 and T1 (middle panel), T3 and T5 (right panel). The high and low groups are divided by the median value of gene signature score. P-value was calculated by multivariate Cox regression. HR, hazard ratio. e, Comparison between proportions of clonal cells in each T cell cluster. 


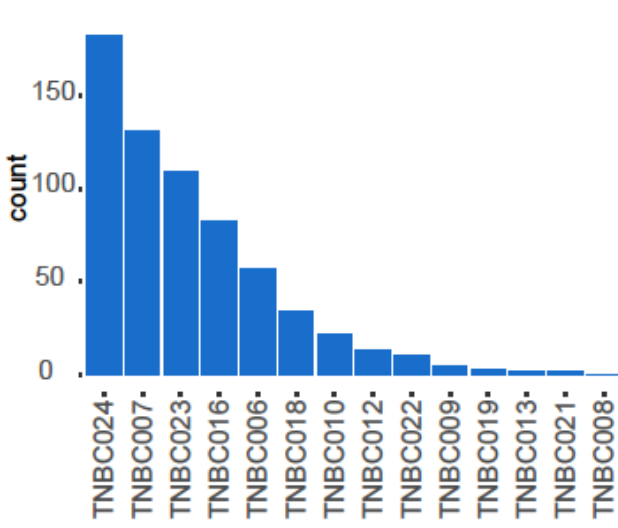

c

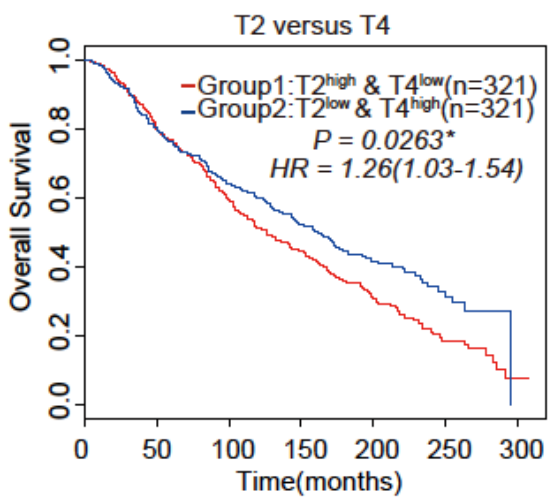

e

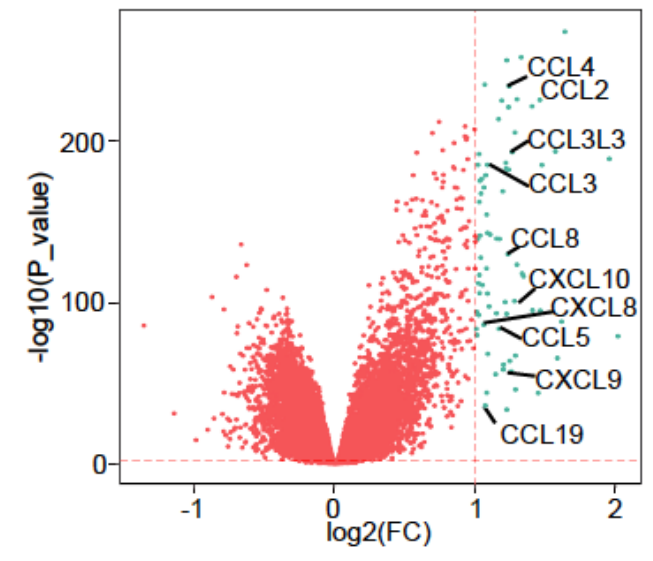

b
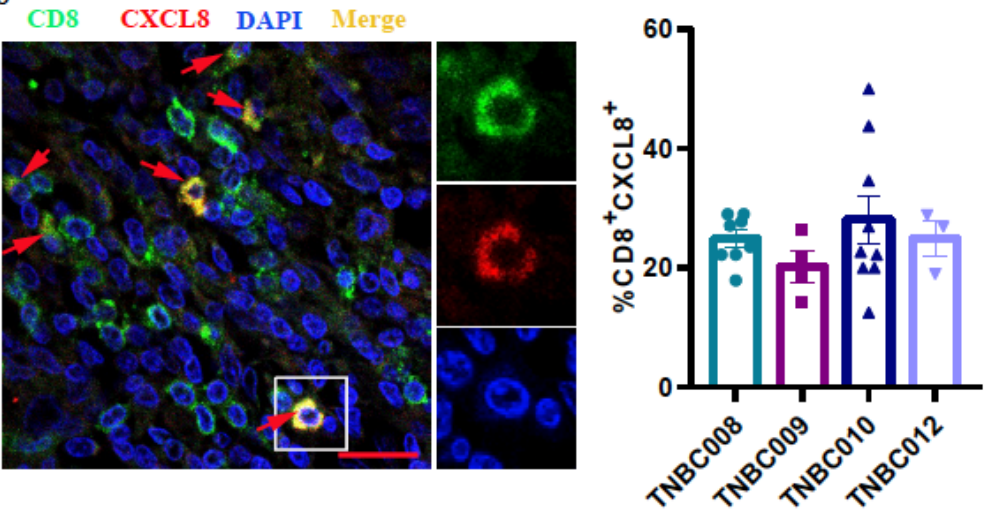

d

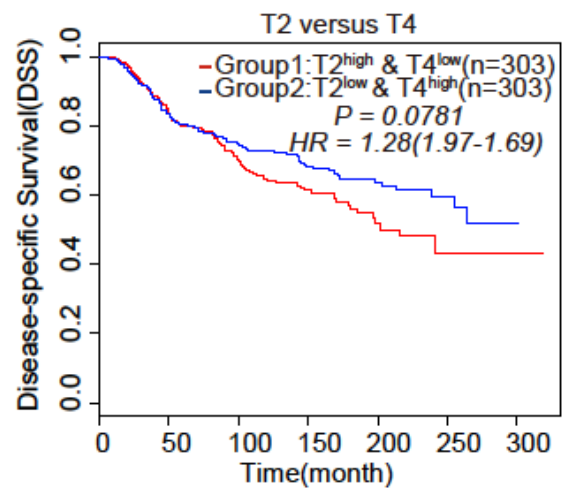

f

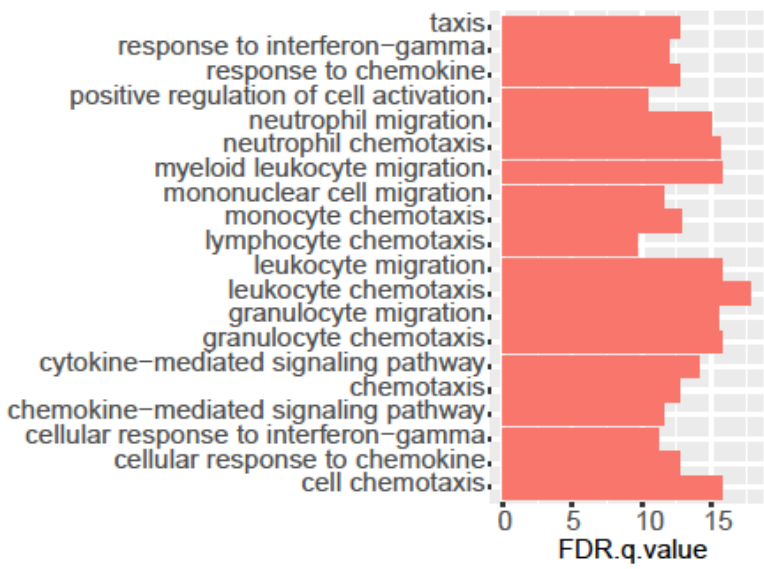

\section{Figure 4}

Analysis of CD8+CXCL8+ T cell subset. a, The distribution of CD8+ CXCL8+ T cell subset (T2) among patients. b, Immunofluorescence showing co-location of CXCL8 and CD8; proportion of CXCL8+ CD8+ cells among CD8+ cells. c, The Kaplan-Meier overall survival curves of METABRIC patients grouped by the gene signature score (see Method) of T2 and T4. The high and low groups are divided by the median value of gene signature score. P-value was calculated by multivariate Cox regression. HR, hazard ratio. $d$, Disease-specific survival (DSS) curve of METABRIC patients grouped by the gene signature score (see Method) of T2 and T4, excluding CXCL8. The high and low groups are divided by the median value of gene signature score. P-value was calculated by multivariate Cox regression. HR, hazard ratio. e, Differential genes between T2highT4low and T4highT2low group from the METABRIC cohort. Each blue 


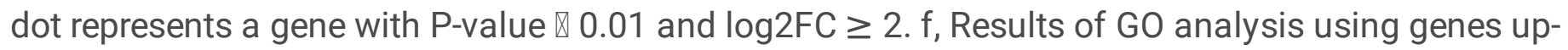
regulated in patients with T2high signature.

Figure 5
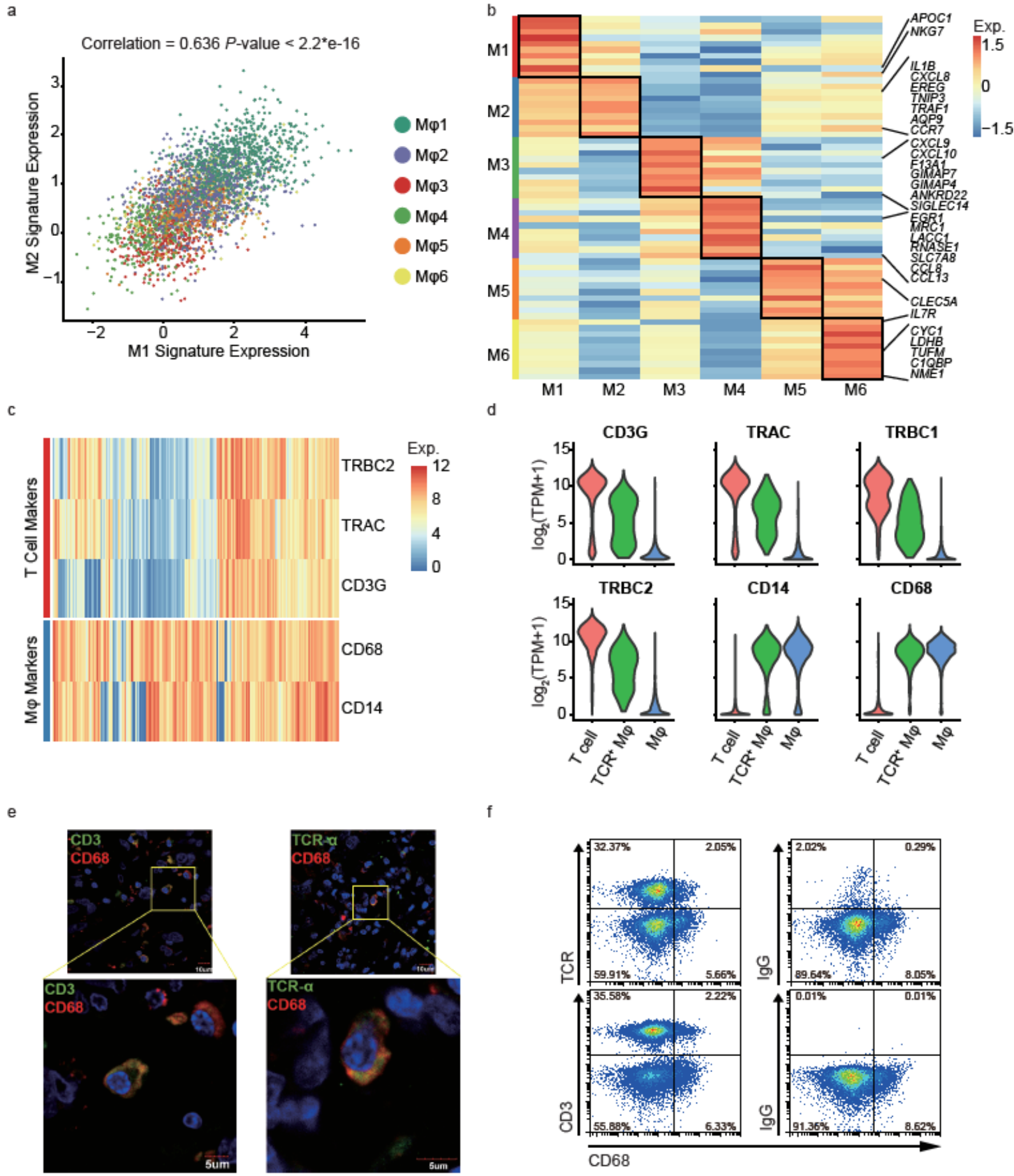

\section{Figure 5}

Characteristics of macrophage clusters and TCR+ macrophages. a, Scatterplot of mean normalized expression (see Method) of M1 and M2 signatures per cell. Each point represents one single cell, colored by clusters. b, Z-score normalized mean expression $(\log 2(T P M+1))$ of representative markers of each 
macrophage cluster. Black boxes highlight the markers for specific cluster. c, The expression $(\log 2(T P M+1))$ of T cell marker (CD3G), TCR genes (TRBC2 and TRAC) and macrophage markers (CD68 and CD14) in TCR+ macrophages $(n=382)$. $d$, Violin plots show the expression $(\log 2(T P M+1))$ of above genes across T cells, TCR+ macrophages and normal (TCR-) macrophages. e, Immunofluorescence proposes the co-expression of TCR/CD3 and CD68 in paraffin section of breast cancer samples. $f, C_{-}$ expression of TCR/CD3 and CD68 revealed by FACS in fresh breast cancer samples.

Figure 6

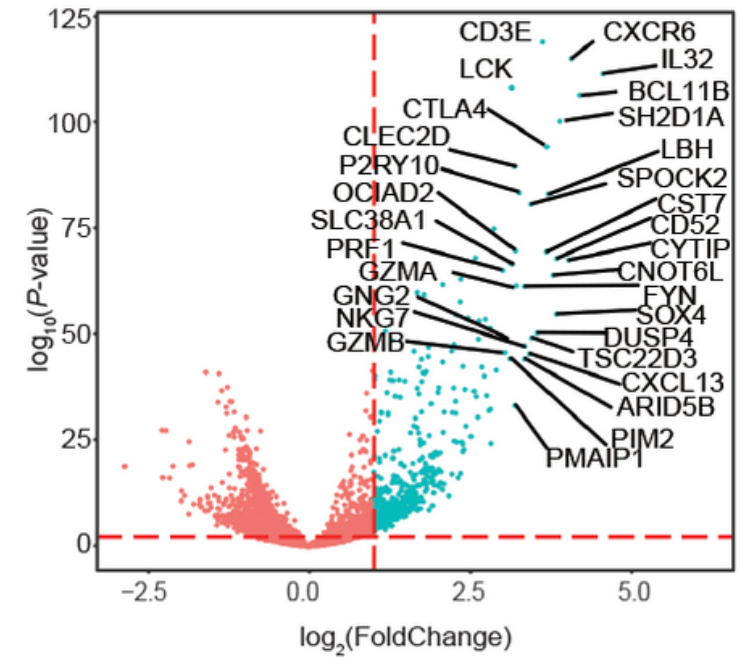

c

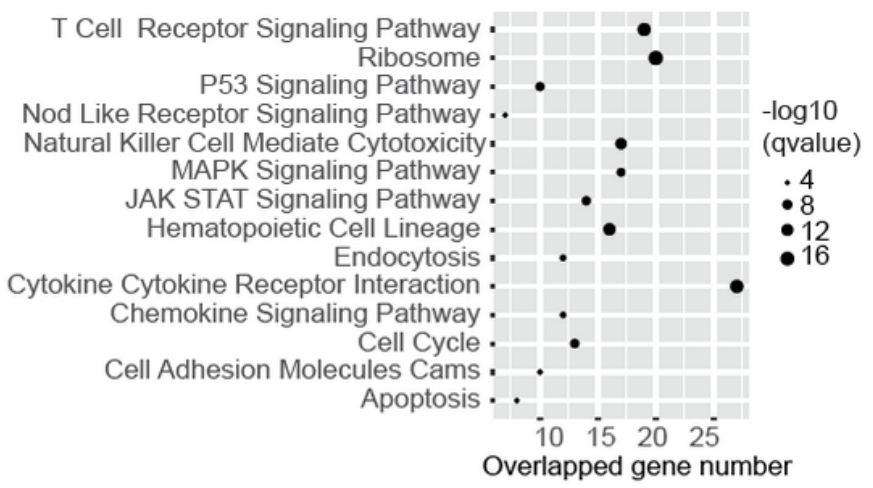

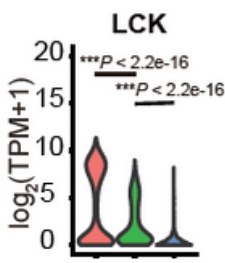

Icos
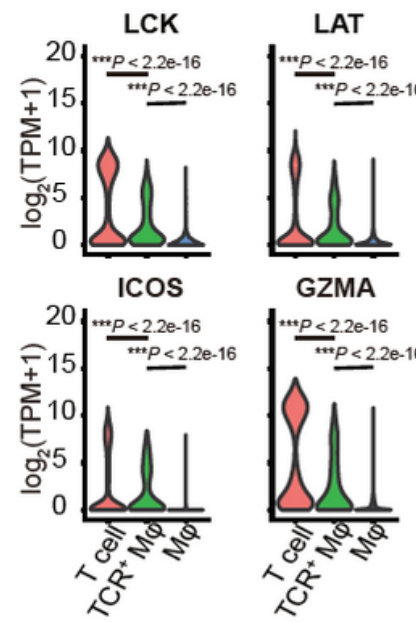

GZMA
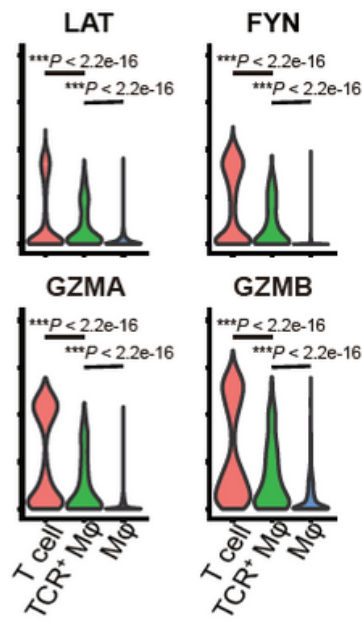

GZMB

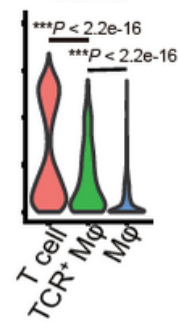

d
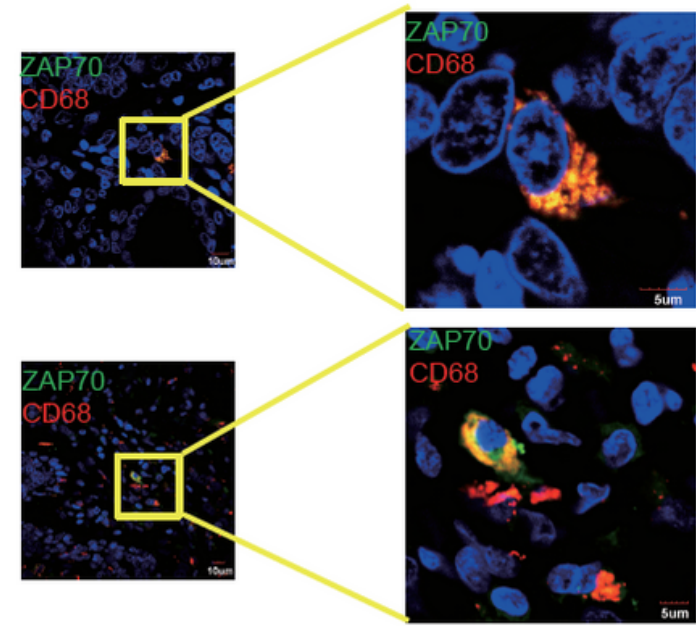

\section{Figure 6}

Activation of TCR signaling pathways. a, Volcano plot shows differentially expressed genes in tumorinfiltrating TCR+ macrophages compared with TCR- macrophages. Each blue dot represents a gene with $P$-value $<0.01$ and $\log 2 F C \geq 1$. Genes with $P$-value $<0.01$ and $\log 2 F C \geq 3$ are marked with gene names.

b, The expression (log2(TPM+1)) of LCK, LAT, FYN, ICOS, GZMA, and GZMB are significantly up-regulated in the TCR+ macrophages comparing to TCR- macrophages. c, Gene set enrichment analysis in KEGG pathway for DEGs with P-value $<0.01$ and $\log 2 F C \geq 1$. Selected pathways with Q-value $<0.01$ are shown in the bubble plot. d, Immunofluorescence shows the co-expression of phosphorylated ZAP-70 and CD68 in paraffin sections. 


\section{Supplementary Files}

This is a list of supplementary files associated with this preprint. Click to download.

- supplemantaryfigures.doc

- TableS1.xIsx

- TableS2.xlsx

- Tables3.xIsx

- TableS4.xlsx

- TableS5.xlsx

- TableS6.xlsx

- TableS7.xlsx

- TableS8.xlsx

- Tables9.xIsx

- TableS10.xlsx 